Revised version, April 22, 2001

\title{
The calibration method for the Mumford-Shah functional and free-discontinuity problems
}

\author{
Giovanni Alberti, Guy Bouchitté, Gianni Dal Maso
}

\begin{abstract}
In this paper we present a minimality criterion for the MumfordShah functional, and more generally for non convex variational integrals on $S B V$ which couple a surface and a bulk term. This method provides short and easy proofs for several minimality results.

Keywords: Mumford-Shah functional, free discontinuity problems, special functions of bounded variation, necessary condition for minimality, calibrations, minimal partitions, gradient flow.

Mathematics Subject Classification (2000): 49K10 (49Q15, 49Q05, 58E12).
\end{abstract}

\section{Introduction}

The Mumford-Shah functional was introduced in [36] and [37] within the context of a variational approach to image segmentation problems (cf. [37] and [31], Chapter 4). In dimension $n$ it can be written as follows

$$
F(u):=\int_{\Omega \backslash S u}|\nabla u|^{2} d x+\alpha \mathcal{H}^{n-1}(S u)+\beta \int_{\Omega}(u-g)^{2} d x
$$

where $\Omega$ is a bounded regular domain in $\mathbf{R}^{n}, g: \Omega \rightarrow[0,1]$ is a given function (input grey level), $\alpha$ and $\beta$ are positive (tuning) parameters, $\mathcal{H}^{n-1}$ is the $(n-1)$ dimensional Hausdorff measure (that is, the usual $(n-1)$-dimensional area in case of subsets of regular hypersurfaces, the length in the most relevant case $n=2$ ). The unknown function $u: \Omega \rightarrow \mathbf{R}$ is regular (say, of class $C^{1}$ ) out of a closed singular set $S u$, whose shape and location are not prescribed. Thus minimizing $F$ means optimizing the function $u$ and the singular set $S u$. Indeed, in the original formulation only the planar case $n=2$ is considered, and the singular set is explicitly viewed as an independent variable, $u$ being smooth on the complement of this set.

While existence results for minimizers of $F$ in dimension two could be proved within the original framework (cf. [37], [18], or [31], Chapter 15), in arbitrary dimension they were first obtained by a different approach outlined by E. De Giorgi (cf. [20]). More precisely, $F$ can be defined for every function $u$ in the space $S B V(\Omega)$ of special functions with bounded variation (see Section 2 for more details, or [6], Chapter 4; this space can be regarded as a sort of the completion of 
piecewise regular functions), upon which it is lower semicontinuous and coercive with respect to the $L^{1}$ topology (cf. [3], or [6, Chapter 6). This immediately yields minimizers of $F$ within this space, which can be proved to be minimizers of the original functional by suitable regularity theorems (cf. [21], see also [6], Chapter 6, for further regularity results). Furthermore, the lower semicontinuity and coercivity results in [3] apply to a larger class of functionals coupling bulk and surface contributions, among which $F$ should be regarded as a prototypical example. Consequently, the $S B V$ setting has been used to model a wide range of problems, from image segmentation, to fractures in brittle materials, to nematic liquid crystals (see [6], Section 4.6, for a survey).

On a mathematical level, one of the most relevant features of the MumfordShah functional is a deep lack of convexity. Hence, not only minimizers may be not unique, but "identifying" them is by no means an easy task, also in terms of efficient algorithms. Clearly, every minimizer $u$ of $F$ must satisfy certain equilibrium conditions - an Euler-Lagrange equation of a sort - which can be obtained by considering different types of infinitesimal variations (see for instance [37] or [6], Section 7.4). Among these we mention the following: $u$ satisfies $\Delta u=\beta(u-g)$ in the complement of the singular set $S u$, the normal derivative of $u$ on $S u$ must vanish (where $S u$ is a regular surface), while the mean curvature of $S u$ multiplied by $\alpha$ is equal to the difference of the energy densities $|\nabla u|^{2}+\beta(u-g)^{2}$ on the two sides of $S u$ (the first two correspond respectively to the Euler-Lagrange equation for the convex functional $\int_{\Omega \backslash K}\left[|\nabla u|^{2}+\beta(u-g)^{2}\right] d x$ and the associated Neumann boundary condition on $\partial K$; together they are equivalent to minimality with prescribed singular set $S u=K)$. However, due to the lack of convexity of $F$, these conditions do not imply minimality - not even local minimality.

In this paper we propose a sufficient condition for minimality, and describe some applications.

We remark that the problem of finding sufficient conditions for minimality already makes sense for a simplified version of $F$ which occurs in the theory of interior regularity for minimizers of $F$, and is obtained by dropping the lower order term in (1.1) and setting for simplicity $\alpha=1$, that is,

$$
F_{0}(u):=\int_{\Omega \backslash S u}|\nabla u|^{2} d x+\mathcal{H}^{n-1}(S u)
$$

For the time being we focus on minimizers of $F_{0}$ with prescribed boundary values (in short, Dirichlet minimizers), and describe the basic idea of this paper, without dwelling on details. More precise definitions and accurate statements will be given in Section 3.

Assuming that $u$ and $S u$ are sufficiently regular, let $u^{+}$and $u^{-}$denote the limits of $u$ on the two sides of $S u$, so that $u^{+}>u^{-}$, and let $\nu_{u}$ be the unit normal to $S u$ pointing from the side of $u^{-}$to that of $u^{+}$; the complete graph of $u$ is the boundary of the subgraph of $u$ (the set of all points $(x, t) \in \Omega \times \mathbf{R}$ such that $t \leq u(x))$, oriented by the inner normal $\nu_{\Gamma u}$. Thus $\Gamma u$ consists of the union of 
the usual graph of $u$ and an additional part given by all segments with endpoints $\left(x, u^{-}(x)\right)$ and $\left(x, u^{+}(x)\right)$, with $x$ ranging in $S u$; see Figure 1 (cf. also Remark $2.11)$.
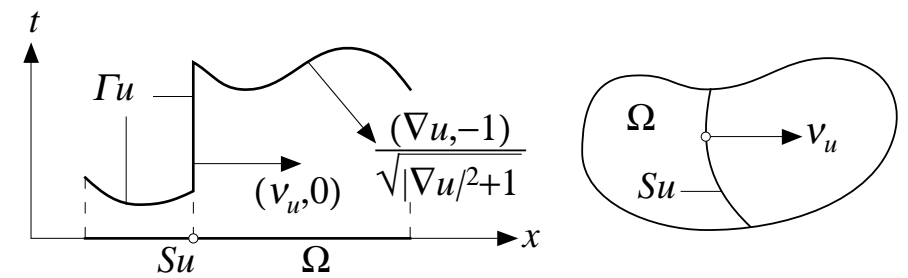

FiguRE 1

We consider now the vectorfields $\phi=\left(\phi^{x}, \phi^{t}\right)$ on $\Omega \times \mathbf{R}$ such that, for every function $u, F_{0}(u)$ is larger than or equal to the flux of $\phi$ through $\Gamma u$, that is

$$
F_{0}(u) \geq \int_{\Gamma u} \phi \cdot \nu_{\Gamma u} d \mathcal{H}^{n}
$$

Taking into account (1.2) and the fact that the flux of $\phi$ through $\Gamma u$ is given by (cf. Figure 1)

$$
\int_{\Omega}\left[\phi^{x}(x, u) \cdot \nabla u-\phi^{t}(x, u)\right] d x+\int_{S u}\left[\int_{u^{-}}^{u^{+}} \phi^{x}(x, t) d t\right] \cdot \nu_{u} d \mathcal{H}^{n-1}
$$

we see that inequality (1.3) is satisfied if

$$
\phi^{x}(x, u) \cdot \nabla u-\phi^{t}(x, u) \leq|\nabla u|^{2} \quad \text { for every } x \in \Omega \backslash S u
$$

and

$$
\left|\int_{u^{-}}^{u^{+}} \phi^{x}(x, t) d t\right| \leq 1 \quad \text { for every } x \in S u .
$$

The first inequality holds for every $u$ if $\phi$ satisfies $\phi^{x}(x, t) \cdot \xi-\phi^{t}(x, t) \leq|\xi|^{2}$ for every point $(x, t)$ and for every vector $\xi$, which can be equivalently restated as

(a) $\left|\phi^{x}(x, t)\right|^{2} \leq 4 \phi^{t}(x, t)$ for $x \in \Omega, t \in \mathbf{R}$, while the second inequality is satisfied if

(b) $\left|\int_{t_{1}}^{t_{2}} \phi^{x}(x, t) d t\right| \leq 1$ for $x \in \Omega, t_{1}, t_{2} \in \mathbf{R}$.

Moreover, one easily checks that equality holds in (1.3) for a particular $u$ if (and only if)

(a') $\phi^{x}(x, u(x))=2 \nabla u(x)$ and $\phi^{t}(x, u(x))=|\nabla u(x)|^{2}$ for $x \in \Omega \backslash S u$,

(b') $\int_{u^{-}(x)}^{u^{+}(x)} \phi^{x}(x, t) d t=\nu_{u}(x)$ for $x \in S u$. 
Let now be given a function $u$, and assume that there exists a vectorfield $\phi$ which is divergence-free and satisfies assumptions (a), (b), (a'), and (b') above. Then for every function $v$ which agrees with $u$ on the boundary of $\Omega$ we have

$$
F_{0}(v) \geq \int_{\Gamma v} \phi \cdot \nu_{\Gamma v} d \mathcal{H}^{n}=\int_{\Gamma u} \phi \cdot \nu_{\Gamma u} d \mathcal{H}^{n}=F_{0}(u)
$$

where the first equality follows from the divergence theorem, since $\phi$ is divergencefree and $\Gamma u$ and $\Gamma v$ have the same boundary. Hence the existence of such a vectorfield $\phi$ implies the minimality of $u$. In Section 3 we give a more precise version of this result, and extend it to minimizers of $F$ and other functionals (cf. Theorems 3.4 and 3.10). In particular, for minimal partitions we recover the principle of paired calibrations introduced in [34] and [11] (in fact, a slight generalization of it - cf. Theorem 3.11 and Remark 3.12).

We call $\phi$ a calibration (for $u$ ) by analogy with the corresponding theory for minimal surfaces: in that setting, a vectorfield $\phi$ is said to calibrate an oriented hypersurface $S$ (with boundary) if it agrees on $S$ with the normal vectorfield, is divergence-free, and satisfies $|\phi| \leq 1$ everywhere; the existence of a calibration implies that $S$ minimizes the area among all oriented hypersurfaces with the same boundary, and the proof is just one line, as (1.5) above (we refer the reader to [32] for detailed references and a review of many results). Calibrations have also been defined for general integrals on oriented surfaces (or currents) of any dimension and codimension (cf. [24], notice that in this general framework they are closed differential form rather than divergence-free vectorfields). Moreover, since every variational integral of the form $\int_{\Omega} f(x, u, \nabla u) d x$ with $u: \Omega \rightarrow \mathbf{R}^{k}$ and $f$ polyconvex in $\nabla u$ can be re-written as a one-homogeneous convex integral on the graph of $u$, the theory of calibrations can be adapted also to these problems, yielding the equivalent of null-lagrangians.

However, this geometric interpretation does not cover free-discontinuity problems, because functionals of Mumford-Shah type cannot be written as integral over the (complete) graph of $u$. Indeed, the novelty of our approach consists in introducing suitable non-local constraints (namely, condition (b)) to define the class of admissible calibrations.

Once the general principle of calibrations is given, two fundamental questions arise: does every minimizer admit a calibration? And how can we recover it? In other words, we have given a sufficient condition for minimality, but we do not know if it is actually fulfilled by any minimizer, and, which is even more relevant in applications, we do not know how to verify it, that is, how to construct a calibration.

To discuss the first question, we begin by recalling the situation of minimal surfaces. The basic idea behind calibrations is that the area functional admits a natural extension from regular oriented surfaces (with fixed boundary) to all normal currents (with the same boundary), which is nothing else but the mass. But now we have a convex functional on an affine space, and hence minimum points 
are exactly characterized by the fact that the subdifferential of the functional contains the zero element; in the specific case, this turns out to be equivalent to the existence of a calibration with Borel coefficients (cf. [24], Proposition 4.10(3)). Thus a given surface admits a calibration of a sort if and only if it minimizes the area (the mass) among all normal currents with same boundary. However, in codimension (and dimension) larger than one, minimizing the area in the class of surfaces, or even integral currents, does not necessarily imply minimizing the area in the class of normal currents. In other words, the infima of the area functional on the two classes may not coincide: counterexamples were given, for instance, in [33] and [40].

The situation of the Mumford-Shah functional is somehow similar. Let $\mathcal{G}_{0}$ be the class of all vectorfields $\phi$ on $\Omega \times \mathbf{R}$ which satisfy assumptions (a) and (b) above; since for every function $u$ we can find $\phi \in \mathcal{G}_{0}$ which also satisfies assumptions (a') and (b'), we have the identity

$$
F_{0}(u)=\sup _{\phi \in \mathcal{G}_{0}} \int_{\Gamma u} \phi \cdot \nu_{\Gamma u} d \mathcal{H}^{n}=\sup _{\phi \in \mathcal{G}_{0}} \int_{\Omega \times \mathbf{R}} \phi \cdot D 1_{u}
$$

where $D 1_{u}$ is the (distributional) derivative of the the characteristic function $1_{u}$ of the subgraph of $u$. Thus $F_{0}(u)=G\left(1_{u}\right)$, where $G(v)$ is defined as the supremum of $\int_{\Omega \times \mathbf{R}} \phi \cdot D v$ over all $\phi \in \mathcal{G}_{0}$ for every function $v \in B V_{\text {loc }}(\Omega \times \mathbf{R})$. Since $G$ is a convex functional on the affine space $X_{u}$ of all functions $v$ which agree with $1_{u}$ on the boundary, $1_{u}$ minimizes $G$ if and only if the subdifferential of $G$ at $1_{u}$ contains the zero element, condition which is roughly equivalent to the existence of a calibration for $u$. It was proved in [15] for the one-dimensional case $n=1$ that the function $1_{u}$ minimizes $G$ whenever $u$ minimizes $F_{0}$; in other words, the infimum of $G$ on $X_{u}$ agrees with the infimum of $F_{0}$ (among all functions with same boundary values as $u$ ), thus proving that every minimizer of $F_{0}$ can be calibrated (in some sense). It is not known if the same result holds in higher dimension.

The discussion of this point may benefit from being set in a more abstract framework. We can indeed summarize the calibration method as follows: given a nonconvex function(al) $F$ on a certain space $X$, we embed $X$ into a convex space $\tilde{X}$ so that $F$ agrees with a convex function $\tilde{F}$ on $\tilde{X}$. Thus the minimality on a point $x \in X$ with respect to $\tilde{F}$ can be characterized via the subdifferential of $\tilde{F}$ at $x$; this yields a sufficient condition for the minimality with respect to $F$, conditions that is also necessary when (and only when) the infima of $\tilde{F}$ on $X$ and $\tilde{X}$ agree. As pointed out in [10], it is always possible to construct the convex space $\tilde{X}$ and the function $\tilde{F}$ so that the two infima agree, but the problem is that checking the condition of minimality derived from this abstract construction may be neither simpler nor more feasible than a direct verification of minimality.

Passing to the second question mentioned above, we notice that an abstract result which guarantees the existence of calibrations would nevertheless provide no solution to the problem of construction. As a matter of fact, we do not know of any general method of construction, not even for minimal surfaces. Instead, we 
have collected in Sections 4 and 5 many examples of calibrations for $F_{0}$ and $F$, and gathered some helpful remarks and observations. Despite a lack of a general recipe, the calibration method provides short and easy proofs of some natural minimality result; among these we recall the following ones:

(1) every harmonic function is a Dirichlet minimizer of $\int_{\Omega}|\nabla u|^{2} d x$, and is also a Dirichlet minimizer of $F_{0}$ when the gradient is sufficiently small (Paragraph 4.10);

(2) a function which is constant on each element of a minimal partition of the domain is a Dirichlet minimizer of $F_{0}$ when the values are sufficiently far apart from each other; in particular this applies to the so-called triple junction (Paragraphs 4.14 and 4.16);

(3) every solution of the equation $-\Delta u+\beta(u-g)=0$ with Neumann boundary conditions is a minimizer of $\int_{\Omega}\left[|\nabla u|^{2}+\beta(u-g)^{2}\right] d x$, and also of $F$ for large $\beta$ (Paragraph 5.4);

(4) if $g$ is the characteristic function of a regular set, then $u:=g$ minimizes $F$ for large $\beta$ (Paragraph 5.5).

Notice that (3) and (4) give a strong indication that for initial data with smooth singular sets the gradient flow associated with $F_{0}$ in the $L^{2}$ metric (which can be defined via time discretization, cf. [27]) leaves the singular set still, at least for small times, and agrees with the heat flow elsewhere (cf. [26] and Remarks 5.12, $5.15,5.16)$.

We conclude this introduction with a few remarks. A long-standing conjecture on the Mumford-Shah functional is that the "cracktip", namely the function $u$ on the plane given in polar coordinates by $u:=\sqrt{2 \rho / \pi} \sin (\theta / 2)$, with $-\pi<\theta \leq \pi$, (or, equivalently, the imaginary part of $\sqrt{2 z / \pi}$ with a cut along the negative real axis) is a Dirichlet minimizer of the homogeneous Mumford-Shah functional $F_{0}$ on every bounded open subset of $\mathbf{R}^{2}$. This conjecture has been recently proved in [9], but so far no calibration has been found for this minimizer.

As shown in [12] in the case of minimal partitions, the calibration method can be numerically implemented to get rigorous lower bounds for the value of minima, to disprove the minimality of a given configuration, or viceversa to get an idea of what the calibration (if it exists) should look like. It is not clear if an efficient numerical implementation for the Mumford-Shah functional is feasible.

The theory presented in this paper is limited to scalar functions. To develop a similar theory for functionals on $\mathbf{R}^{k}$-valued maps $u$, one should replace divergencefree vectorfields by closed $n$-forms on $\Omega \times \mathbf{R}^{k}$, which act on the graphs of the maps $u$, viewed as (suitably defined) $n$-surfaces in $\Omega \times \mathbf{R}^{k}$.

This paper is organized as follows. In Section 2 we recall the basic notation about finite perimeter sets and the space $S B V$, which is indeed the natural setting for our theory (however, under most regards the unfamiliar reader can just replace the word $S B V$ with "smooth out of a piecewise smooth singular set", and "finite perimeter" with "piecewise smooth boundary"). In Section 3 we expand the idea 
outlined above and develop the theory of calibrations for minimizers of $F_{0}$ and $F$, with or without prescribed boundary values. Then we discuss the extension to more general functionals, and the connection with paired calibrations. Sections 4 and 5 are devoted to examples and applications. Finally, the appendix contains the proofs of some technical results stated in Section 2.

Some of the results contained in this paper were announced in [2] and [16]. Further applications can be found in [17] and [30].

ACKNowledgements. - The first and third authors have been partially supported by MURST through the projects "Equazioni Differenziali e Calcolo delle Variazioni (1997)" and "Calcolo delle Variazioni (2000)". This research was initiated while the first author was visiting the University of Toulon, and subsequently developed during a stay at the Max Planck Institute for Mathematics in the Sciences in Leipzig. Several people contributed, with discussions and remarks, to the final shape of this paper; among them, we would like to thank in particular Antonin Chambolle and Massimo Gobbino.

\section{Notation and preliminary results}

Throughout this paper, sets and functions are always assumed to be Borel measurable, and we do not identify functions which agree almost everywhere. A vectorfield on a subset $E$ of $\mathbf{R}^{n}$ is any map from $E$ into $\mathbf{R}^{n}$. The divergence of a vectorfield is always intended in the sense of distributions (in the interior of its domain); in particular we say that $\phi$ is divergence-free to mean that its divergence vanishes.

The letter $\Omega$ denotes a (possibly unbounded) open subset of $\mathbf{R}^{n}, \mathbf{S}^{n-1}$ is the unit sphere in $\mathbf{R}^{n}, \mathcal{H}^{k}$ stands for the $k$-dimensional Hausdorff measure (which agrees with the usual $k$-dimensional volume on every regular surface of dimension $k$ ), and $\mathcal{L}^{n}$ is the $n$-dimensional Lebesgue measure; when the integration is done with respect to Lebesgue measure, we always write $d x$ instead of $d \mathcal{L}^{n}$. The restriction of any Borel measure $\mu$ to a set $E$ is denoted by $\mu\llcorner E$, while $g \cdot \mu$ is the (vector) measure canonically associated with any $\mu$-summable (vector) function $g$. Thus $\mu\left\llcorner E=1_{E} \cdot \mu\right.$ where $1_{E}$ is the characteristic function of $E$, namely the function which is equal to 1 on $E$ and to 0 elsewhere. $\|\cdot\|_{p}$ denotes the norm in the space $L^{p}$.

A (vector) function $f$ on $\mathbf{R}^{n}$ has approximate limit $a$ at $x$, and we write $\underset{y \rightarrow x}{\operatorname{ap} \lim } f(y)=a$, if

$$
\lim _{r \rightarrow 0} \frac{1}{r^{n}} \int_{B(x, r)}|f(y)-a| d y=0
$$

The same definition applies to functions defined on a subset $E$ of $\mathbf{R}^{n}$, provided that $B(x, r)$ is replaced by $E \cap B(x, r)$. Notice that this definition slightly differs from the more usual one, which is expressed in term of the density at $x$ of the 
pre-images of neighbourhoods of $a$ (cf. [22], Section 1.7.2, and [6], Definition 3.63); however, these two notions agree for locally bounded functions.

We recall now some notation and basic facts about finite perimeter sets, $B V$ and $S B V$ functions; for a more precise definitions and a detailed account of the results we refer to [6], Chapters 3 and 4 (for the theory of $B V$ functions, see also [22], Chapter 5).

A real function $u$ on an open set $\Omega \subset \mathbf{R}^{n}$ has bounded variation in $\Omega$, and we write $u \in B V(\Omega)$, if it belongs to $L^{1}(\Omega)$ and the distributional derivative $D u$ is (represented by) a bounded vector measure on $\Omega$. The integral of an $\mathbf{R}^{n}$-valued function $f$ with respect to the measure derivative $D u$ is denoted by $\int_{\Omega} f \cdot D u$. Every $u \in B V(\Omega)$ is almost everywhere differentiable in the approximate (or measure theoretic) sense, and the corresponding approximate gradient $\nabla u$ agrees with the density of the measure $D u$ with respect to Lebesgue measure. If $\Omega$ has Lipschitz boundary, then $u$ admits a trace on the boundary (in the approximate sense), which we still denote by $u$, and which belongs to $L_{\text {loc }}^{1}\left(\partial \Omega, \mathcal{H}^{n-1}\right)$.

The singular set $S u$ is the set of all points where $u$ admits no approximate limit; $S u$ has Hausdorff dimension (less than or) equal to $n-1$, and more precisely it is rectifiable (of dimension $n-1$ ), which means that it can be covered, up to an $\mathcal{H}^{n-1}$-negligible subset, by countably many hypersurfaces of class $C^{1}$ (these sets are sometimes called "countably $\left(\mathcal{H}^{n-1}, n-1\right)$-rectifiable", cf. [23], Section 3.2.14). We recall that, for every rectifiable set, the approximate unit normal, and the corresponding approximate tangent hyperplane, are well-defined at $\mathcal{H}^{n-1}$-almost every point (and do not depend on the choice of the covering). The approximate unit normal to $S u$ at $x$ is denoted by $\nu_{u}(x)$. At $\mathcal{H}^{n-1}$-almost every $x \in S u$ there exist the approximate limits $u^{+}(x)$ and $u^{-}(x)$ of $u$ on the two sides of $S u$ (more precisely, the approximate limits of the restriction of $u$ to the two half-spaces defined by the approximate tangent hyperplane at $x$ ), and we arrange so that $u^{+}(x)>u^{-}(x)$, and $\nu_{u}(x)$ is pointing from the side of $u^{-}(x)$ to the one of $u^{+}(x)$.

The measure $D u$ can be canonically decomposed as the sum of three mutually orthogonal measures: the Lebesgue part $\nabla u \cdot \mathcal{L}^{n}$, the jump part $\left(u^{+}-u^{-}\right) \nu_{u}$. $\mathcal{H}^{n-1}\llcorner S u$, and a remainder, called Cantor part, which is singular but does not charge any $\mathcal{H}^{n-1}$-finite set.

The space $S B V(\Omega)$ of special functions of bounded variation is given by all functions $u \in B V(\Omega)$ for which the Cantor part of the derivative vanishes, i.e.,

$$
D u=\nabla u \cdot \mathcal{L}^{n}+\left(u^{+}-u^{-}\right) \nu_{u} \cdot \mathcal{H}^{n-1}\llcorner S u .
$$

A subset $E$ of $\Omega$ has finite perimeter (in $\Omega$ ) if the distributional derivative $D 1_{E}$ of its characteristic function is a bounded vector measure on $\Omega$. In this case, the measure theoretic boundary $\partial_{*} E$ is the singular set of $1_{E}$, while the inner normal $\nu_{\partial_{*} E}$ is the associated normal vectorfield $\nu_{1_{E}}$. Both the Lebesgue and the Cantor parts of $D 1_{E}$ vanish, i.e.,

$$
D 1_{E}=\nu_{\partial_{*} E} \cdot \mathcal{H}^{n-1}\left\llcorner\partial_{*} E\right.
$$




\section{A general form of the divergence theorem}

When looking for calibrations for a given function, it is often convenient to consider also vectorfields which are not regular. In doing so, however, we face some technical difficulties.

First of all, the first identity in (1.5) depends on the divergence theorem, and may not hold when $\phi$ is divergence-free (in the sense of distribution) but not continuous, because the flux of such a vectorfield through a given surface is not well-defined. To solve this problem, we must assume a certain regularity in $\phi$.

Definition 2.1. - We say that a vectorfield $\phi$ on a subset $E$ of $\mathbf{R}^{n}$ is approximately regular if it is bounded, and for every Lipschitz hypersurface $M$ in $\mathbf{R}^{n}$ there holds

$$
\underset{y \rightarrow x}{\operatorname{ap} \lim }\left[\phi(y) \cdot \nu_{M}(x)\right]=\phi(x) \cdot \nu_{M}(x) \quad \text { for } \mathcal{H}^{n-1} \text {-a.e. } x \in M \cap E,
$$

where $\nu_{M}(x)$ denotes the (unit) normal to $M$ at $x$.

REMARK 2.2. - If $\phi$ is approximately regular, then (2.4) can be extended to every rectifiable set $M, \nu_{M}$ being now understood in the approximate sense. If $\phi$ admits traces $\phi^{+}$and $\phi^{-}$on the two sides of $M$ (defined in the same way of the traces $u^{+}$and $u^{-}$), then (2.4) is equivalent to the compatibility condition

$$
\phi \cdot \nu_{M}=\phi^{+} \cdot \nu_{M}=\phi^{-} \cdot \nu_{M} \quad \mathcal{H}^{n-1} \text {-a.e. in } M \cap E
$$

which links the pointwise values of $\phi$ on $M$ with the values of the traces.

In particular, if $\phi$ is (approximately) continuous $\mathcal{H}^{n-1}$-almost everywhere on $E$, then it is approximately regular. Similarly, if $\phi$ is (approximately) continuous on the complement of a rectifiable set $S$, then $\phi$ is approximately regular if and only if (2.4) holds for $M:=S$.

REMARK 2.3. - If $\phi$ has the special form $\phi:=(0, \ldots, 0, \psi)$ where $\psi=$ $\psi\left(x_{1}, \ldots, x_{n}\right)$ is a bounded real function which is continuous in the variable $x_{n}$, then $\phi$ is approximately regular. Take indeed a Lipschitz surface $M$, and let $M_{0}$ be the subset of all points $x \in M$ such that the $n$-th component of $\nu_{M}(x)$ vanishes. Then equality (2.4) obviously holds for all $x \in M_{0}$. To prove that it also holds for $\mathcal{H}^{n-1}$-a.e. point in $M \backslash M_{0}$ it suffices to notice that $\psi$ is approximately continuous in the complement of a set of type $N \times \mathbf{R}$, where $N$ is a $\mathcal{L}^{n-1}$-negligible subset of $\mathbf{R}^{n-1}$. Thus $\phi$ is approximately continuous at all points except those in $N \times \mathbf{R}$, which form an $\mathcal{H}^{n-1}$-negligible subset of $M \backslash M_{0}$ by the area formula (see [23], Theorem 3.2.22, or, for a slightly less general statement, [6], Theorem 2.91).

We can now state a refined version of the classical divergence theorem (the proof is postponed to the appendix). 
Lemma 2.4. - Let $\Omega$ be an open set in $\mathbf{R}^{n}$ with Lipschitz boundary, $\phi$ an approximately regular vectorfield on $\bar{\Omega}$, and $u$ a function in $B V(\Omega)$. Assume moreover that $\operatorname{div} \phi \in L^{\infty}(\Omega)$ and $u \phi \in L^{1}\left(\partial \Omega, \mathcal{H}^{n-1}\right)$. Then

$$
\int_{\Omega} \phi \cdot D u=-\int_{\Omega} u \operatorname{div} \phi d x-\int_{\partial \Omega} u \phi \cdot \nu_{\partial \Omega} d \mathcal{H}^{n-1}
$$

where $\nu_{\partial \Omega}$ is the inner unit normal to $\partial \Omega$, and in the last integral $u$ stands for the trace of $u$ on $\partial \Omega$.

Notice that the condition $u \phi \in L^{1}\left(\partial \Omega, \mathcal{H}^{n-1}\right)$ is always satisfied when $\partial \Omega$ is bounded, because in this case the trace of $u$ belongs to $L^{1}\left(\partial \Omega, \mathcal{H}^{n-1}\right)$.

REMARK 2.5. - As shown in [7], for a Borel vectorfield $\phi$ with divergence in $L^{1}$ it is possible to give a functional definition of the trace of the normal component of $\phi$ on any Lipschitz surface $M$, and precisely as a continuous extension of the trace operator for regular vectorfields. When $M$ is the boundary (of some set $\Omega$ ), this notion of trace automatically satisfies the divergence theorem (in the sense that formula (2.6) holds for every function $u$ of class $C_{c}^{1}$ ), but since modifying $\phi$ in a Lebesgue-negligible set affects neither this trace nor the distributional divergence, one can easily produce examples where the trace does not agree with the normal component of $\phi$ on $M$. Thus Lemma 2.4 shows implicitly that this is never the case when $\phi$ is approximately regular. One may wonder if every vectorfield with divergence in $L^{\infty}$ agrees, up to a Lebesgue-negligible set, with an approximately regular one. Unfortunately the answer is negative, even for divergence-free vectorfields (cf. [7], example after Proposition 2.1).

Going back to the first identity in (1.5), we remark that verifying that a vectorfield $\phi$ is divergence-free is relatively easy when $\phi$ is of class $C^{1}$ because the distributional divergence agrees with the classical one, which can be explicitly computed. If $\phi$ is piecewise $C^{1}$, the task is slightly more difficult, and can be carried out in many concrete cases (see Sections 4 and 5) with the help of the following lemma (the proof is postponed to the appendix).

LEMMA 2.6. - Let $\phi$ be a bounded vectorfield on an open set $\Omega \subset \mathbf{R}^{n}$, and assume that there exist a closed set $S$ and a function $f \in L_{\mathrm{loc}}^{1}(\Omega)$ such that $\operatorname{div} \phi=f$ in the sense of distributions on $\Omega \backslash S$. Then the identity $\operatorname{div} \phi=f$ holds also on $\Omega$ if $S$ can be written as $S:=S_{0} \cup S_{1}$, with $S_{0}$ an $\mathcal{H}^{n-1}$-negligible closed set and $S_{1}$ a (possibly disconnected) Lipschitz hypersurface, and $\phi$ satisfies (2.4) for $M:=S_{1}$ and $E:=\Omega$.

REMARK 2.7. - The point of this lemma is roughly the following: since the divergence is a first order differential operator, $\operatorname{div} \phi$ cannot "charge" any set of codimension larger than 1 , and therefore $S_{0}$ can be safely removed. On the other hand, the part of $\operatorname{div} \phi$ supported on the hypersurface $M$ is given by the difference of the traces (whenever defined) of the normal components of $\phi$ on the two sides 
of $M$, which happens to vanish if (2.4) holds, and then we are allowed to neglect $S_{1}$ too.

REMARK 2.8. - Lemma 2.6 will be often applied in the following forms.

(a) Suppose that $\phi$ is a bounded vectorfield on $\bar{\Omega}$, continuous on $\bar{\Omega} \backslash\left(S_{0} \cup S_{1}\right)$, and divergence-free on $\Omega \backslash\left(S_{0} \cup S_{1}\right)$, with $S_{0}$, and $S_{1}$ given as above. If $\phi$ satisfies (2.4) with $M=S_{1}$, then $\phi$ is approximately regular on $\bar{\Omega}$ and divergence-free on $\Omega$ (cf. Remark 2.2).

(b) Let be given, for $j=1, \ldots, m$, pairwise disjoint Lipschitz open sets $\Omega_{j}$ whose closures cover $\bar{\Omega}$, and approximately regular, divergence-free vectorfields $\phi_{j}$ on $\bar{\Omega}_{j}$. Let $\phi$ be any vectorfield on $\bar{\Omega}$ which agrees at any point with one of the $\phi_{i}$ (hence it is uniquely determined at least on the union of all $\Omega_{i}$ ). Then $\phi$ is approximately regular and divergence-free provided that the vectorfields $\phi_{i}$ satisfy the compatibility conditions

$$
\phi_{i} \cdot \nu_{\partial \Omega_{i}}=\phi_{j} \cdot \nu_{\partial \Omega_{j}} \quad \mathcal{H}^{n-1} \text {-a.e. on } \partial \Omega_{i} \cap \partial \Omega_{j},
$$

which are equivalent to the compatibility condition (2.5) for $\phi$.

\section{The complete graph of an $S B V$ function}

We fix now some notation and state some results which are more specific to this paper. In the following $\Omega$ is a fixed bounded open subset of $\mathbf{R}^{n}$ with Lipschitz boundary, and $\nu_{\partial \Omega}$ is its inner unit normal. The letter $x$ usually denotes the variable in $\Omega$ (or $\mathbf{R}^{n}$ ), while $t$ is the variable in $\mathbf{R} ; U$ is an open subset of $\Omega \times \mathbf{R}$, with Lipschitz boundary whose closure can be written as

$$
\bar{U}:=\left\{(x, t) \in \bar{\Omega} \times \mathbf{R}: \tau_{1}(x) \leq t \leq \tau_{2}(x)\right\},
$$

where the functions $\tau_{1}, \tau_{2}: \bar{\Omega} \rightarrow[-\infty,+\infty]$ satisfy $\tau_{1}<\tau_{2}$. The letter $\phi$ denotes a bounded vectorfield defined on (a subset of) $\mathbf{R}^{n} \times \mathbf{R}$, with components $\phi^{x} \in \mathbf{R}^{n}$ and $\phi^{t} \in \mathbf{R}$. Notice that $\operatorname{div} \phi=\operatorname{div}_{x} \phi^{x}+\partial_{t} \phi^{t}$, where $\operatorname{div}_{x}$ is the (distributional) divergence with respect to $x$ and $\partial_{t}$ the (distributional) derivative with respect to $t$.

Definition 2.9. - For every function $u \in B V(\Omega)$, let $1_{u}$ be the characteristic function of the subgraph of $u$ in $\Omega \times \mathbf{R}$, namely $1_{u}(x, t):=1$ for $t \leq u(x)$ and $1_{u}(x, t):=0$ for $t>u(x)$. The complete graph of $u$, denoted by $\Gamma u$, is the measure theoretic boundary of the subgraph of $u$, i.e., the singular set of $1_{u}$.

Since the subgraph of $u$ has finite perimeter in $\Omega \times \mathbf{R}$ (see, e.g., [29], Proposition 1.4), the definition of $\Gamma u$ is well-posed. Moreover $D 1_{u}=\nu_{\Gamma u} \cdot \mathcal{H}^{n}\llcorner\Gamma u$ (cf. (2.3)), where $\nu_{\Gamma u}$ is the inner unit normal of the subgraph of $u$. Therefore the flux through $\Gamma u$ of any vectorfield $\phi$ on $\Omega \times \mathbf{R}$ is given by the integration of $\phi$ with respect to the vector measure $D 1_{u}$, that is

$$
\int_{\Gamma u} \phi \cdot \nu_{\Gamma u} d \mathcal{H}^{n}=\int_{\Omega \times \mathbf{R}} \phi \cdot D 1_{u} .
$$


An alternative way to compute this flux is given by the following lemma (the proof is postponed to the appendix).

LEMmA 2.10. - Let $u$ be a function in $S B V(\Omega)$ and let $\phi$ be a vectorfield defined at least on $\Gamma u$. Then

$$
\begin{aligned}
\int_{\Gamma u} \phi \cdot \nu_{\Gamma u} d \mathcal{H}^{n}= & \int_{\Omega}\left[\phi^{x}(x, u) \cdot \nabla u-\phi^{t}(x, u)\right] d x \\
& +\int_{S u}\left[\int_{u^{-}}^{u^{+}} \phi^{x}(x, t) d t\right] \cdot \nu_{u} d \mathcal{H}^{n-1}
\end{aligned}
$$

where $u, u^{ \pm}, \nabla u$, and $\nu_{u}$ are always computed at $x$.

REMARK 2.11. - Formula (2.9) corresponds to a decomposition of the derivative of $1_{u}$, or, better, to a decomposition of the complete graph $\Gamma u$ as union (up to $\mathcal{H}^{n}$-negligible sets) of a "regular" part - namely the set of all points $(x, u(x))$ such that $u$ is approximately continuous at $x$, and has approximate gradient $\nabla u(x)$ - and a "vertical" part - namely the set of all points $(x, t)$ with $x \in S u$ and $t \in\left(u^{-}(x), u^{+}(x)\right)$. Note that for a general $B V$ function there would be an additional subset of $\Gamma u$, corresponding to the Cantor part of $D u$.

The following version of the divergence theorem (cf. Lemma 2.4) yields the first equality in (1.5) (the proof is postponed to the appendix).

Lemma 2.12. - Let be given two functions $u$ and $v$ in $B V(\Omega)$ whose complete graphs lie in $\bar{U}$, and an approximately regular vectorfield $\phi$ on $\bar{U}$ which is divergence-free in $U$. Then

$$
\begin{aligned}
\int_{\Gamma u} \phi \cdot \nu_{\Gamma u} d \mathcal{H}^{n} & -\int_{\Gamma v} \phi \cdot \nu_{\Gamma v} d \mathcal{H}^{n}= \\
& =\int_{\partial \Omega}\left[\int_{u}^{v} \phi^{x}(x, t) d t\right] \cdot \nu_{\partial \Omega} d \mathcal{H}^{n-1}
\end{aligned}
$$

(where, in the last integral, $u$ and $v$ stand for the traces on $\partial \Omega$ ).

\section{Calibrations for free discontinuity problems}

In this section we introduce a calibration principle for a wide class of free discontinuity problems, expanding the basic idea explained in the introduction. We begin with the case of the Mumford-Shah functional, with or without the lower order term, and then we consider more general functionals, possibly with discontinuous integrands, which include some interesting functionals considered in minimal partition problems.

Throughout this section $\Omega$ is a bounded open subset of $\mathbf{R}^{n}$ with Lipschitz boundary, $U$ is an open set with Lipschitz boundary contained in $\Omega \times \mathbf{R}$ which 
satisfies (2.7); $u$ always denotes a function in $S B V(\Omega)$. The functionals $F(u)$ and $F_{0}(u)$ are given in (1.1) and (1.2), respectively, where $S u$ and $\nabla u$ are now defined as in Section $2, \alpha>0$ and $\beta \geq 0$ are fixed constants, and $g$ belongs to $L^{\infty}(\Omega)$. Note that the functional $F_{0}$ is the particular case of $F$ corresponding to $\alpha=1$ and $\beta=0$. In the following definition we fix some terminology about minimizers of $F$ (which also applies to any other functional on $S B V$ ).

Definition 3.1. - We say that a function $u$ is an (absolute) minimizer of $F$ if $F(u) \leq F(v)$ for all $v \in S B V(\Omega)$, while $u$ is a Dirichlet minimizer if $F(u) \leq F(v)$ for all $v \in S B V(\Omega)$ with same trace on $\partial \Omega$ as $u$ (that is, with same boundary values as $u)$. We say that $u$ is a $\bar{U}$-minimizer if the complete graph of $u$ is contained in $\bar{U}$ and $F(u) \leq F(v)$ for all $v \in S B V(\Omega)$ with complete graph contained in $\bar{U}$, while $u$ is a $\bar{U}$-Dirichlet minimizer if we add the requirement that the competing functions $v$ have the same boundary values as $u$.

\section{Calibrations for the Mumford-Shah functional}

We generalize the idea described in the introduction and provide sufficient conditions for $\bar{U}$-minimality and $\bar{U}$-Dirichlet minimality with respect to the MumfordShah functional $F$ (or $F_{0}$ ). We begin with the following key lemma.

Lemma 3.2. - Let $F$ be defined as in (1.1) for some $\alpha>0, \beta \geq 0$, and let $\phi$ be a vectorfield on $\bar{U}$ which satisfies the following assumptions:

(a) $\phi^{t}(x, t) \geq \frac{1}{4}\left|\phi^{x}(x, t)\right|^{2}-\beta(t-g)^{2}$ for $\mathcal{L}^{n}$-a.e. $x \in \Omega$ and every $t \in\left[\tau_{1}, \tau_{2}\right]$,

(b) $\left|\int_{t_{1}}^{t_{2}} \phi^{x}(x, t) d t\right| \leq \alpha$ for $\mathcal{H}^{n-1}$-a.e. $x \in \Omega$ and every $t_{1}, t_{2} \in\left[\tau_{1}, \tau_{2}\right]$,

where the functions $\tau_{1}$ and $\tau_{2}$ are defined by (2.7) and, like $g$, are computed at $x$. Then for every $u$ such that $\Gamma u \subset \bar{U}$ we have

$$
F(u) \geq \int_{\Gamma u} \phi \cdot \nu_{\Gamma u} d \mathcal{H}^{n}
$$

Moreover, equality holds in (3.1) for a given $u$ if and only if

(a') $\phi^{x}(x, u)=2 \nabla u$ and $\phi^{t}(x, u)=|\nabla u|^{2}-\beta(u-g)^{2}$ for $\mathcal{L}^{n}$-a.e. $x \in \Omega$,

(b') $\int_{u^{-}}^{u^{+}} \phi^{x}(x, t) d t=\alpha \nu_{u}$ for $\mathcal{H}^{n-1}$-a.e. $x \in S u$,

where $u, u^{ \pm}, \nabla u, \nu_{u}$, and $g$ are always computed at $x$.

Proof. Take $u$ such that $\Gamma u \subset \bar{U}$. We recall that by Lemma 2.10

$$
\begin{aligned}
\int_{\Gamma u} \phi \cdot \nu_{\Gamma u} d \mathcal{H}^{n}= & \int_{\Omega}\left[\phi^{x}(x, u) \cdot \nabla u-\phi^{t}(x, u)\right] d x \\
& +\int_{S u}\left[\int_{u^{-}}^{u^{+}} \phi^{x}(x, t) d t\right] \cdot \nu_{u} d \mathcal{H}^{n-1} .
\end{aligned}
$$


It is an elementary fact that for every $\xi, \eta \in \mathbf{R}^{n}$ we have $\xi \cdot \eta-\frac{1}{4}|\xi|^{2} \leq|\eta|^{2}$, and equality holds if and only if $\xi=2 \eta$. Hence, setting $\xi:=\phi^{x}(x, u)$ and $\eta:=\nabla u$, and taking (a) into account, we obtain that, $\mathcal{L}^{n}$-a.e. on $\Omega$,

$$
\begin{aligned}
\phi^{x}(x, u) \cdot \nabla u-\phi^{t}(x, u) & \leq \phi^{x}(x, u) \cdot \nabla u-\frac{1}{4}\left|\phi^{x}(x, u)\right|^{2}+\beta(u-g)^{2} \\
& \leq|\nabla u|^{2}+\beta(u-g)^{2}
\end{aligned}
$$

and consequently

$$
\int_{\Omega}\left[\phi^{x}(x, u) \cdot \nabla u-\phi^{t}(x, u)\right] d x \leq \int_{\Omega}\left[|\nabla u|^{2}+\beta(u-g)^{2}\right] d x .
$$

Moreover, equality holds in (3.3) if and only if $\phi^{x}(x, u)=2 \nabla u$ and $\phi^{t}(x, u)=$ $\frac{1}{4}\left|\phi^{x}(x, u)\right|^{2}-\beta(u-g)^{2}=|\nabla u|^{2}-\beta(u-g)^{2}$ for a.e. $x \in \Omega$, which is (a').

As for the second integral in the right-hand side of (3.2), condition (b) above implies

$$
\left[\int_{u^{-}}^{u^{+}} \phi^{x}(x, t) d t\right] \cdot \nu_{u} \leq\left|\int_{u^{-}}^{u^{+}} \phi^{x}(x, t) d t\right| \leq \alpha \quad \mathcal{H}^{n-1} \text {-a.e. on } S u
$$

and then

$$
\int_{S u}\left[\int_{u^{-}}^{u^{+}} \phi^{x}(x, t) d t\right] \cdot \nu_{u} d \mathcal{H}^{n-1} \leq \alpha \mathcal{H}^{n-1}(S u) .
$$

Moreover it is clear that equality holds in (3.4) if and only if (b') is satisfied.

Inequality (3.1) follows now from (3.2), (3.3), and (3.4), as well as the rest of the statement.

REMARK 3.3. - Let $\mathcal{G}$ be the class of all vectorfields $\phi$ on $\Omega \times \mathbf{R}$, not necessarily bounded, which satisfy assumptions (a) and (b) of Lemma 3.2 with $U:=\Omega \times \mathbf{R}$. It can be easily proved that for every $u$ in $S B V(\Omega)$ there exists $\phi \in \mathcal{G}$ which satisfies assumptions (a') and (b') for $u$, so that equality holds in (3.1). Starting from this, one can also find vectorfields $\phi \in \mathcal{G}$ of class $C_{c}^{1}$ such that the value of the flux in the right-hand side of (3.1) is arbitrarily close to $F(u)$. Taking (2.8) into account, we can thus prove that for all $u \in S B V(\Omega)$ there holds (cf. (1.6))

$$
F(u)=\sup _{\phi \in \mathcal{G} \cap C_{c}^{1}} \int_{\Gamma u} \phi \cdot \nu_{\Gamma u} d \mathcal{H}^{n}=\sup _{\phi \in \mathcal{G} \cap C_{c}^{1}} \int_{\Omega \times \mathbf{R}} \phi \cdot D 1_{u}
$$

Moreover one can show that for any function $u$ which is in $B V(\Omega)$, but not in $S B V(\Omega)$, the last two terms in (3.5) are equal to $+\infty$. Since every integral of the form $\int_{\Omega \times \mathbf{R}} \phi \cdot D 1_{u}$, with $\phi$ of class $C_{c}^{1}$ on $\Omega \times \mathbf{R}$, is continuous with respect to the weak* topology of $B V(\Omega)$, formula (3.5) shows that the Mumford-Shah functional $F$, extended to $+\infty$ to the rest of $B V(\Omega)$, is weak* lower semicontinuous. The 
same argument can be easily applied to the general functionals considered in the next subsection, providing another proof of the well-known compactness and semicontinuity results in $S B V$ due to L. Ambrosio (see [3], or [6], Sections 4.1 and 5.4).

TheOREM 3.4. - Let $u$ be a function with complete graph contained in $\bar{U}$, and assume that there exists an approximately regular vectorfield $\phi$ on $\bar{U}$ which is divergence-free on $U$ and satisfies assumptions (a), (b), (a'), and (b') of Lemma 3.2. Then $u$ is a Dirichlet $\bar{U}$-minimizer of $F$. If, in addition, the normal component of $\phi$ at the boundary of $\Omega \times \mathbf{R}$ vanishes, namely

$$
\phi^{x} \cdot \nu_{\partial \Omega}=0 \quad \mathcal{H}^{n} \text {-a.e. on }(\partial \Omega \times \mathbf{R}) \cap \partial U
$$

then $u$ is also an absolute $\bar{U}$-minimizer of $F$.

Proof. Let $v$ be a function in $S B V(\Omega)$ such that $v=u$ on $\partial \Omega$ and $\Gamma v \subset \bar{U}$. Then

$$
F(v) \geq \int_{\Gamma v} \phi \cdot \nu_{\Gamma v} d \mathcal{H}^{n}=\int_{\Gamma u} \phi \cdot \nu_{\Gamma u} d \mathcal{H}^{n}=F(u)
$$

Here, the first inequality and the last equality follow from Lemma 3.2, while the first equality follows from Lemma 2.12. We have thus proved that $u$ is a Dirichlet $\bar{U}$-minimizer of $F$. Viceversa, assuming (3.6) we obtain that the first equality in (3.7) holds even if the traces of $v$ and $u$ on $\partial \Omega$ differ, which proves that $u$ is an absolute $\bar{U}$-minimizer of $F$.

Definition 3.5. - We call the vectorfield $\phi$ in the first part of Theorem 3.4 $a$ Dirichlet calibration for $u$ on $\bar{U}$ (with respect to $F$ ). If $\phi$ satisfies the additional assumption (3.6), then we call it an absolute calibration.

When $U:=\Omega \times \mathbf{R}$ we omit to write it. When it is clear from the context, we may also omit to specify the functional, the set $U$, and whether the calibration is Dirichlet or absolute, and simply say that $\phi$ is a calibration for $u$, or that $\phi$ calibrates $u$.

REMARK 3.6. - If $\phi$ is an absolute calibration for $u$, then it is also an absolute calibration for every other minimizer. Indeed, if $F(v)=F(u)$, the first inequality in (3.7) must be an equality, and by Lemma 3.2 this means that $\phi$ satisfies assumptions (a') and (b') for $v$ too. Similarly, if $\phi$ is a Dirichlet calibration for $u$, then it is also a Dirichlet calibration for any other Dirichlet minimizer with the same boundary values as $u$.

This fact can be sometimes used to prove that the minimizer is unique. For instance, if $\phi$ calibrates a function $u$ with a negligible singular set (i.e., $\mathcal{H}^{n-1}(S u)=0$ ), and the inequality in assumption (b) is always strict, then we deduce that assumption (b') can only be satisfied by functions with negligible singular sets, and therefore all minimizers should have this property. But on this class the functional $F$ is strictly convex (for $\beta>0$, and even for $\beta=0$ in case of 
Dirichlet minimizers), and therefore the minimizer must be unique (see Remarks 4.7, 4.11, 5.2, and Paragraphs 5.4 and 5.5).

REMARK 3.7. - The functional $F_{0}$ in (1.2) is obtained by setting $\alpha:=1$ and $\beta:=0$ in the definition of $F$. In this specific case, assumptions (a), (b), (a'), and (b') in Lemma 3.2 become

(a) $\phi^{t}(x, t) \geq \frac{1}{4}\left|\phi^{x}(x, t)\right|^{2}$ for $\mathcal{L}^{n}$-a.e. $x \in \Omega$ and every $t \in\left[\tau_{1}, \tau_{2}\right]$,

(b) $\left|\int_{t_{1}}^{t_{2}} \phi^{x}(x, t) d t\right| \leq 1$ for $\mathcal{H}^{n-1}$-a.e. $x \in \Omega$ and every $t_{1}, t_{2} \in\left[\tau_{1}, \tau_{2}\right]$,

(a') $\phi^{x}(x, u)=2 \nabla u$ and $\phi^{t}(x, u)=|\nabla u|^{2}$ for $\mathcal{L}^{n}$-a.e. $x \in \Omega$,

(b') $\int_{u^{-}}^{u^{+}} \phi^{x}(x, t) d t=\nu_{u}$ for $\mathcal{H}^{n-1}$-a.e. $x \in S u$.

REMARK 3.8. - It must be noticed that, given a boundary value $w$ in $L^{1}\left(\partial \Omega, \mathcal{H}^{n-1}\right)$, the Dirichlet problem

$$
\min \left\{F_{0}(u): u \in S B V(\Omega), u=w \mathcal{H}^{n-1} \text {-a.e. on } \partial \Omega, \Gamma u \subset \bar{U}\right\}
$$

may not have a solution, even for a very regular $w$, due to a lack of continuity of the trace operator on $S B V(\Omega)$. Therefore problem (3.8) is usually replaced by the relaxed problem

$$
\min \left\{F_{0}(u)+\mathcal{H}^{n-1}(\{x \in \partial \Omega: u(x) \neq w(x)\}): u \in S B V(\Omega), \Gamma u \subset \bar{U}\right\}
$$

(where in the second term $u$ denotes, as usual, the trace of $u$ on $\partial \Omega$ ). A variant of the standard lower semicontinuity and compactness theorems in $S B V(\Omega)$ (see [14] or [8]) shows that problem (3.9) has always a solution.

The calibration method applies to problem (3.9), too. In this case calibrations are approximately regular vectorfields $\phi$ on $\bar{\Omega} \times \mathbf{R}$ which are divergence-free, and satisfy conditions (a), (b), (a'), (b') of Remark 3.7 and, in addition, the following two conditions (which may be viewed as extensions of (b) and (b') to the boundary):

$$
\begin{aligned}
& \text { (c) }\left|\int_{w}^{s} \phi^{x}(x, t) d t\right| \leq 1 \text { for } \mathcal{H}^{n-1} \text {-a.e. } x \in \partial \Omega \text { and every } s \in\left[\tau_{1}, \tau_{2}\right], \\
& \text { (c') } \int_{w}^{u} \phi^{x}(x, t) d t=\nu_{\partial \Omega} \text { for } \mathcal{H}^{n-1} \text {-a.e. } x \in \partial \Omega \text { with } u(x) \neq w(x),
\end{aligned}
$$

where $u$ and $w$ are computed at $x$, as well as $\tau_{1}$ and $\tau_{2}$. Indeed, arguing as in Lemma 3.2 we obtain

$$
\begin{aligned}
F_{0}(v)+ & \mathcal{H}^{n-1}(\{x \in \partial \Omega: v(x) \neq w(x)\}) \geq \\
& \geq \int_{\Gamma v} \phi \cdot \nu_{\Gamma v} d \mathcal{H}^{n}+\int_{\partial \Omega}\left[\int_{w}^{v} \phi^{x}(x, t) d t\right] \cdot \nu_{\partial \Omega} d \mathcal{H}^{n-1}
\end{aligned}
$$

for every $v \in S B V(\Omega)$, with equality for $v=u$, moreover the right hand side of (3.10) does not depend on $v$ (apply Lemma 2.12 taking into account that $\phi$ is divergence free). 


\section{Calibrations for general functionals}

The method of calibrations can be easily adapted to a larger class of functionals on $S B V(\Omega)$. Take indeed

$$
\Psi(u):=\int_{\Omega} f(x, u, \nabla u) d x+\int_{S u} \psi\left(x, u^{-}, u^{+}, \nu_{u}\right) d \mathcal{H}^{n-1},
$$

where $f: \Omega \times \mathbf{R} \times \mathbf{R}^{n} \rightarrow[0,+\infty]$ and $\psi: \Omega \times \mathbf{R} \times \mathbf{R} \times \mathbf{S}^{n-1} \rightarrow[0,+\infty]$. We refer to [3] for general conditions on $f$ and $\psi$ which imply the lower semicontinuity of the functional (3.11) and guarantee the existence of minimizers. However, lower semicontinuity is irrelevant for the calibration method.

Let $f^{*}$ and $\partial_{\xi} f$ denote the convex conjugate and the subdifferential of $f$ with respect to the last variable. We recall that the subdifferential of a function $g$ : $\mathbf{R}^{n} \rightarrow[0,+\infty]$ at the point $\xi \in \mathbf{R}^{n}$ is defined as the set of vectors $\eta \in \mathbf{R}^{n}$ such that $g(\xi)+\eta \cdot(\zeta-\xi) \leq g(\zeta)$ for every $\zeta \in \mathbf{R}^{n}$. It is well known that for every $\xi, \eta \in \mathbf{R}^{n}$ we have the inequality $\xi \cdot \eta-g^{*}(\eta) \leq g(\xi)$, and that equality holds if and only if $\eta \in \partial_{\xi} g(\xi)$. Using these properties we obtain the following variant of Lemma 3.2, whose proof is omitted.

LEMMA 3.9. - Let $\phi$ be a vectorfield on $\bar{U}$ which satisfies the following assumptions:

(a) $\phi^{t}(x, t) \geq f^{*}\left(x, t, \phi^{x}(x, t)\right)$ for $\mathcal{L}^{n}$-a.e. $x \in \Omega$ and every $t \in\left[\tau_{1}, \tau_{2}\right]$,

(b) $\left[\int_{t_{1}}^{t_{2}} \phi^{x}(x, t) d t\right] \cdot \nu \leq \psi\left(x, t_{1}, t_{2}, \nu\right)$ for $\mathcal{H}^{n-1}$-a.e. $x \in \Omega$, every $\nu \in \mathbf{S}^{n-1}$, and every $t_{1}<t_{2}$ in $\left[\tau_{1}, \tau_{2}\right]$.

Then for every $u$ with complete graph contained in $\bar{U}$ we have

$$
\Psi(u) \geq \int_{\Gamma u} \phi \cdot \nu_{\Gamma u} d \mathcal{H}^{n} .
$$

Moreover, equality holds in (3.12) for a given $u$ if and only if

(a') $\phi^{x}(x, u) \in \partial_{\xi} f(x, u, \nabla u)$ and $\phi^{t}(x, u)=f^{*}\left(x, u, \phi^{x}(x, u)\right)$ for $\mathcal{L}^{n}$-a.e. $x \in \Omega$,

(b') $\left[\int_{u^{-}}^{u^{+}} \phi^{x}(x, t) d t\right] \cdot \nu_{u}=\psi\left(x, u^{-}, u^{+}, \nu_{u}\right)$ for $\mathcal{H}^{n-1}$-a.e. $x \in S u$,

where $u, u^{ \pm}, \nabla u$, and $\nu_{u}$ are always computed at $x$.

Proceeding as in the previous subsection, one can prove the following analogue of Theorem 3.4.

TheORem 3.10. - Let $u$ be a function with graph contained in $\bar{U}$. Assume that there exists an approximately regular vectorfield $\phi$ on $\bar{U}$ which is divergencefree and satisfies assumptions (a), (b), (a'), and (b') of Lemma 3.9. Then u is a Dirichlet $\bar{U}$-minimizer of $\Psi$. If, in addition, the normal component of $\phi$ on the boundary of $\Omega \times \mathbf{R}$ vanishes, i.e., (3.6) holds, then $u$ is also an absolute $\bar{U}$-minimizer of $\Psi$. 


\section{Calibrations for minimal partitions}

Besides $F$ and $F_{0}$, interesting examples of functionals of the form (3.11) arise in different variants of the minimal partition problem. Let us consider the case where the number $m$ of the elements of the partition is prescribed. To formulate the problem, we fix real numbers $a_{1}<\cdots<a_{m}$ in an arbitrary way, and consider only functions $u$ in the class $B V\left(\Omega,\left\{a_{i}\right\}\right)$ of all $u \in B V(\Omega)$ which take only these prescribed values. The corresponding level sets $A_{i}:=\left\{u=a_{i}\right\}$, sometimes called phases, form a partition $\Omega$; for $i<j$, the set $S_{i j}$ of all $x \in S u$ such that $u^{-}(x)=a_{i}$ and $u^{+}(x)=a_{j}$ is called the interface between the phases $A_{i}$ and $A_{j}$, and is oriented by the normal $\nu_{i j}$ pointing from $A_{i}$ to $A_{j}$ (hence $\nu_{i j}=\nu_{u}$ ).

We consider functionals of the form

$$
\mathcal{F}\left(A_{1}, \ldots, A_{m}\right)=\sum_{i<j} \int_{S_{i j}} \psi_{i j}\left(x, \nu_{i j}\right) d \mathcal{H}^{n-1},
$$

where $\psi_{i j}: \Omega \times \mathbf{S}^{n-1} \rightarrow[0,+\infty]$. Notice that the weights $\psi_{i j}$ may depend on the point at which two phases meet and on the normal to the interface at that point. A partition $\left(A_{1}, \ldots, A_{m}\right)$ is said to be a Dirichlet minimizer of $\mathcal{F}$ if it minimizes $\mathcal{F}$ among all partitions $\left(B_{1}, \ldots, B_{m}\right)$ such that, for every $i$, the characteristic functions of $A_{i}$ and $B_{i}$ have the same trace on $\partial \Omega$. Notice that all these notions do not depend on the particular choice of the numbers $a_{1}, \ldots, a_{m}$.

We now define a functional $\Psi$ of type (3.11) by setting

$$
\begin{aligned}
f(x, t, \xi) & := \begin{cases}0 & \text { if } t \in\left\{a_{i}\right\} \text { for some } i \text { and } \xi=0, \\
+\infty & \text { otherwise }\end{cases} \\
\psi\left(x, t_{1}, t_{2}, \nu\right) & := \begin{cases}\psi_{i j}(x, \nu) & \text { if } t_{1}=a_{i} \text { and } t_{2}=a_{j} \text { for some } i<j \\
+\infty & \text { otherwise }\end{cases}
\end{aligned}
$$

Since every function $u$ in $B V\left(\Omega,\left\{a_{i}\right\}\right)$ belongs to $S B V(\Omega)$, and $\nabla u=0 \mathcal{L}^{n}$-a.e. in $\Omega$, one easily checks that $\Psi(u)$ is finite only if $u$ belongs to $B V\left(\Omega,\left\{a_{i}\right\}\right)$, and in this case $\Psi(u)=\mathcal{F}\left(A_{1}, \ldots, A_{m}\right)$, where $\left(A_{1}, \ldots, A_{m}\right)$ is the partition associated with $u$. Hence a partition $\left(A_{1}, \ldots, A_{m}\right)$ is a Dirichlet minimizer for $\mathcal{F}$ if and only if the corresponding function $u$ is a Dirichlet minimizer for $\Psi$, which according to Theorem 3.10 is implied by the existence of a calibration.

For this particular choice of $\Psi$, a calibration for $u \in B V\left(\Omega,\left\{a_{i}\right\}\right)$ is an approximately regular vectorfield $\phi$ on $\bar{\Omega} \times \mathbf{R}$ which is divergence-free and satisfies the following properties (cf. Lemma 3.9):

(a) $\phi^{t}\left(x, a_{i}\right) \geq 0$ for $\mathcal{L}^{n}$-a.e. $x \in \Omega$ and every $i$,

(b) $\left[\int_{a_{i}}^{a_{j}} \phi^{x}(x, t) d t\right] \cdot \nu \leq \psi_{i j}(x, \nu)$ for $\mathcal{H}^{n-1}$-a.e. $x \in \Omega$ and every $\nu \in \mathbf{S}^{n-1}$ and $i<j$,

(a') $\phi^{t}\left(x, a_{i}\right)=0$ for $\mathcal{L}^{n}$-a.e. $x \in A_{i}$ and every $i$, 
(b') $\left[\int_{a_{i}}^{a_{j}} \phi^{x}(x, t) d t\right] \cdot \nu_{i j}=\psi_{i j}\left(x, \nu_{i j}\right) \mathcal{H}^{n-1}$-a.e. on $S_{i j}$ for every $i<j$.

These calibrations can be re-written in a different and more interesting way: let us set, for $i=1, \ldots, m, x \in \bar{\Omega}$,

$$
\phi_{i}(x):=\int_{a_{1}}^{a_{i}} \phi^{x}(x, s) d s .
$$

One can check that the vectorfields $\phi_{i}$ are approximately regular on $\bar{\Omega}$ and have divergence in $L^{\infty}(\Omega)$ (more precisely, $\operatorname{div} \phi_{i}(x)=\phi^{x}\left(x, a_{1}\right)-\phi^{x}\left(x, a_{i}\right)$ - apply, e.g., formula (2.6) with $\Omega$ replaced by $\Omega \times\left(a_{1}, a_{i}\right)$, and $u$ any smooth function on $\Omega \times\left(a_{1}, a_{i}\right)$ which depends only on $\left.x\right)$ and satisfy the following properties:

(c) $\operatorname{div} \phi_{i} \geq \operatorname{div} \phi_{j}$ for $\mathcal{L}^{n}$-a.e. $x \in A_{i}$ for every $j \neq i$,

(d) $\left(\phi_{j}-\phi_{i}\right) \cdot \nu \leq \psi_{i j}(x, \nu)$ for $\mathcal{H}^{n-1}$-a.e. $x \in \Omega$ and every $\nu \in \mathbf{S}^{n-1}, i<j$,

(d') $\left(\phi_{j}-\phi_{i}\right) \cdot \nu_{i j}=\psi_{i j}\left(x, \nu_{i j}\right)$ for $\mathcal{H}^{n-1}$-a.e. $x \in S_{i j}$ and every $i<j$.

Conversely, given approximately regular vectorfields $\phi_{i}$ on $\bar{\Omega}$ with divergence in $L^{\infty}(\Omega)$ which satisfy (c), (d), (d'), we define a vectorfield $\phi$ on $\bar{\Omega} \times \mathbf{R}$ as follows: we take smooth non-negative functions $\sigma_{i}$ with support included in $\left(a_{i}, a_{i+1}\right)$ and integral equal to 1 , and set

$$
\phi^{x}(x, t):=\sigma_{i}(t)\left(\phi_{i+1}(x)-\phi_{i}(x)\right) \text { for } x \in \bar{\Omega}, a_{i} \leq t \leq a_{i+1},
$$

then we take $\phi^{t}$ so that

$$
\left\{\begin{array}{lr}
\phi^{t}\left(x, a_{i}\right):=0 & \text { for } x \in A_{i} \\
\partial_{t} \phi^{t}(x, t):=\sigma_{i}(t)\left(\operatorname{div} \phi_{i}(x)-\operatorname{div} \phi_{i+1}(x)\right) & \text { for } x \in \Omega, a_{i} \leq t \leq a_{i+1}
\end{array}\right.
$$

the definition is completed by setting $\phi(x, t):=\phi\left(x, a_{1}\right)$ for $t<a_{1}$ and $\phi(x, t):=$ $\phi\left(x, a_{m}\right)$ for $t>a_{m}$.

Then $\phi$ is divergence free in $\Omega \times \mathbf{R}$ by construction, and one can easily check that it satisfies assumptions (a), (b), (a'), and (b') above. Moreover, since each $\phi_{i}$ is approximately regular on $\bar{\Omega}$, one can verify that $\left(\phi^{x}, 0\right)$ is approximately regular on $\Omega \times \mathbf{R}$, and the same holds for $\left(0, \phi^{t}\right)$ by Remark 2.3 . Hence $\phi$ is approximately regular too.

We have thus proved the following result.

Theorem 3.11. - Let $\left(A_{1}, \ldots, A_{m}\right)$ be a partition of $\Omega$, and assume that there exist approximately regular vectorfields $\phi_{1}, \ldots, \phi_{m}$ on $\bar{\Omega}$ with divergences in $L^{\infty}(\Omega)$ which satisfy assumptions $(\mathrm{c}),(\mathrm{d})$, and (d') above. Then $\left(A_{1}, \ldots, A_{m}\right)$ is a Dirichlet minimizer of the functional $\mathcal{F}$ in (3.13).

REMARK 3.12. - A particularly relevant example of functional of type (3.13) is the "interface size", which is obtained by taking $\psi_{i j} \equiv 1$ for all $i<j$. In this case assumptions (d) and (d') above reduce to 
(d) $\left|\phi_{j}(x)-\phi_{i}(x)\right| \leq 1$ for every $x \in \Omega$ and every $i<j$,

(d') $\phi_{j}(x)-\phi_{i}(x)=\nu_{i j}(x)$ for $\mathcal{H}^{n-1}$-a.e. $x \in S_{i j}$ and every $i<j$.

Calibrations of this type have already been introduced in [34] and [11] as "paired calibrations". More precisely, a paired calibration for a partition $\left(A_{1}, \ldots, A_{m}\right)$ is an ordered $m$-uple of approximately regular vectorfields $\phi_{1}, \ldots, \phi_{m}$ on $\bar{\Omega}$ which are divergence-free and satisfy assumptions (d) and (d') above. We notice that the assumption that the vectorfields $\phi_{i}$ are divergence-free is stronger than (c), and indeed our definition allows in principle for a larger class of calibrations.

Among other applications, in [34] it is shown that in any dimension $n$ the partition of a regular simplex in $\mathbf{R}^{n}$ given by the $n+1$ simplices spanned by one face and the centre is a Dirichlet minimizer of the interface size (and the paired calibration consists simply of $n+1$ constant vectorfields which are orthogonal to the corresponding faces). For $n=3$ this statement was first shown in [38] with a (relatively) long proof. In [11] it is shown that, unlike what happens in dimension 3 , the partition of a hypercube in $\mathbf{R}^{n}, n \geq 4$, given by the $2 n$ simplices spanned by one face and the centre is a Dirichlet minimizer of the interface size. In both papers the theory is also extended to cover more general functionals. For further examples and results, see also [13], [12]; other references are included in the survey [32].

\section{Applications to the homogeneous Mumford-Shah functional}

In this section we give some examples of Dirichlet minimizers of the homogeneous Mumford-Shah functional $F_{0}$. We begin with a few remarks which may be useful when constructing calibrations.

REMARK 4.1. - By a simple truncation argument, to prove that a function $u: \Omega \rightarrow[m, M]$ is a (Dirichlet) minimizer for $F_{0}$ it suffices to show that $F_{0}(u) \leq$ $F_{0}(v)$ for all competitors $v$ such that $m \leq v \leq M$. Thus it is enough to show that $u$ is a Dirichlet $\bar{U}$-minimizer, with $U:=\Omega \times(m, M)$. In the following we often tacitly assume this principle, and construct calibrations in $\bar{\Omega} \times[m, M]$ instead of $\bar{\Omega} \times \mathbf{R}$. Notice, however, that a calibration $\phi$ on $\bar{\Omega} \times[m, M]$ can be extended to $\bar{\Omega} \times \mathbf{R}$ in a rather simple way: it suffices to set $\phi(x, t):=\left(0, \phi^{t}(x, m)\right)$ for $t<m$ and $\phi(x, t):=\left(0, \phi^{t}(x, M)\right)$ for $t>M$ (cf. Remarks 2.3 and 2.8(b)).

The same conclusion holds for $F$ if $g$ satisfies $m \leq g \leq M$ too (but may fail for a functional of the general form (3.11), due to lack of suitable truncations).

REMARK 4.2. - We can construct divergence-free vectorfields on an open set $A \subset \Omega \times \mathbf{R}$ using fibrations of $A$ by graphs of harmonic functions. More precisely, given harmonic functions $u_{\lambda}$ whose graphs are pairwise disjoint and cover $A$, for 
all $(x, t) \in A$ we set

$$
\phi(x, t):=\left(2 \nabla u_{\lambda}(x),\left|\nabla u_{\lambda}(x)\right|^{2}\right),
$$

where $\lambda=\lambda(x, t)$ is taken so that $t=u_{\lambda}(x)$. Thus $\phi$ is a vectorfield on $A$ which, by construction, satisfies assumption (a) of Remark 3.7, and assumption (a') for every $u_{\lambda}$.

We prove that $\phi$ is divergence-free under the additional assumption that the function $u(x, \lambda):=u_{\lambda}(x)$ is of class $C^{1}$ and $\partial_{\lambda} u(x, \lambda) \neq 0$ for every $(x, \lambda)$, which implies that the parameter $\lambda$ can be (locally) chosen so that it depends on $x$ and $t$ in a $C^{1}$ fashion. Then we get

$$
\begin{aligned}
\operatorname{div} \phi & =2 \Delta_{x} u+2 \partial_{\lambda} \nabla_{x} u \cdot \nabla_{x} \lambda+2 \nabla_{x} u \cdot \partial_{\lambda} \nabla_{x} u \partial_{t} \lambda \\
& =2 \partial_{\lambda} \nabla_{x} u \cdot\left(\nabla_{x} \lambda+\nabla_{x} u \partial_{t} \lambda\right) .
\end{aligned}
$$

On the other hand, deriving the identity $u(x, \lambda(x, t))=t$ with respect to $x$ and $t$ we get $\nabla_{x} u+\partial_{\lambda} u \nabla_{x} \lambda=0$ and $\partial_{\lambda} u \partial_{t} \lambda=1$, respectively. This implies that the last factor in (4.2) vanishes, and thus $\phi$ is divergence-free (to make this argument work, we need that $\nabla_{x} u$ is of class $C^{1}$ in $(x, \lambda)$, which can be derived by the fact that each function $u_{\lambda}$ is harmonic).

In Paragraphs 4.6 and 4.10 below we apply this idea by embedding a harmonic function that we intend to calibrate into a family of harmonic functions whose graphs fibrate $A:=\Omega \times(m, M)$, and taking $\phi$ as in (4.2). To show that $\phi$ is a calibration we will have only to verify assumption (b) of Remark 3.7.

REMARK 4.3. - The construction described in the previous remark is a particular case of a classical result about extremal fields of scalar functionals (see, e.g., [1], Section 4). Let be given an open subset $A$ of $\Omega \times \mathbf{R}$ which is covered by a family of pairwise disjoint graphs of solutions $u_{\lambda}$ of the Euler-Lagrange equation associated with the functional $\int_{\Omega} f(x, u, \nabla u) d x$, that is

$$
\operatorname{div}\left(\partial_{\xi} f(x, u, \nabla u)\right)=\partial_{t} f(x, u, \nabla u)
$$

Here $t$ and $\xi$ denote the second and third variable in the argument of $f(x, t, \xi)$, corresponding to $u$ and $\nabla u$, and we assume that $f$ is of class $C^{2}$ in $(t, \xi)$ and convex in $\xi$. For all $(x, t) \in A$ let

$$
\left\{\begin{array}{l}
\phi^{x}(x, t):=\partial_{\xi} f\left(x, u_{\lambda}(x), \nabla u_{\lambda}(x)\right) \\
\phi^{t}(x, t):=\partial_{\xi} f\left(x, u_{\lambda}(x), \nabla u_{\lambda}(x)\right) \cdot \nabla u_{\lambda}(x)-f\left(x, u_{\lambda}(x), \nabla u_{\lambda}(x)\right)
\end{array}\right.
$$

where $\lambda=\lambda(x, t)$ is taken so that $t=u_{\lambda}(x)$. Then $\phi$ satisfies by construction assumption (a) of Lemma 3.9, and assumption (a') for every function $u_{\lambda}$. Moreover one can prove that $\phi$ is divergence-free under the additional assumption that the function $u(x, \lambda):=u_{\lambda}(x)$ is of class $C^{2}$ in $(x, \lambda)$ and $\partial_{\lambda} u \neq 0$ (cf. [1], Theorem 
4.6). If, in addition, $\phi$ satisfies assumption (b) of Lemma 3.9, then it is a calibration for each $u_{\lambda}$ relative to the functional $\Psi$ in (3.11), and hence each $u_{\lambda}$ is a $\bar{U}$-Dirichlet minimizer of $\Psi$ provided that $\bar{U}$ is contained in $A$.

REMARK 4.4. - The construction described in the previous remarks cannot be really used for absolute minimizers, i.e., when no Dirichlet boundary condition is imposed. Indeed, calibrations for absolute minimizers should have vanishing normal component at the boundary of $\Omega \times \mathbf{R}$ (see Theorem 3.10), and this holds true for the vectorfield $\phi$ in (4.3) if and only if the functions $u_{\lambda}$ satisfy the natural boundary condition $\partial_{\xi} f\left(x, u_{\lambda}(x), \nabla u_{\lambda}(x)\right) \cdot \nu_{\partial \Omega}(x)=0$ for $x \in \partial \Omega$. But this means that all $u_{\lambda}$ solve the Neumann problem associated with the functional $\int_{\Omega} f(x, u, \nabla u) d x$, while in general we cannot expect a one-parameter family of solutions for such a problem. For instance, if this functional is strictly convex (and this is indeed the case for the regular part of the Mumford-Shah functional $F$ when $\beta>0$ ), then the associated Neumann problem admits at most one solution.

REMARK 4.5. - For $n=1$ and $\Omega=(a, b)$, the equation $\operatorname{div} \phi=0$ on $\Omega \times \mathbf{R}$, coupled with the identity $\phi^{t}=\frac{1}{4}\left(\phi^{x}\right)^{2}$, reduces to the first order equation

$$
\partial_{x} \phi^{x}+\frac{1}{2} \phi^{x} \partial_{t} \phi^{x}=0 \text {. }
$$

It easily follows from the method of characteristics that $\phi$ is a $C^{1}$ solution of (4.4) in $\Omega \times \mathbf{R}$ if and only if every level set $\left\{\phi^{x}=s\right\}$ is composed of straight lines with slope $s / 2$ (intersected with $\Omega \times \mathbf{R}$ ). In other words, for $n=1$ all $C^{1}$ divergence-free vectorfields $\phi$ on $\Omega \times \mathbf{R}$ which satisfy $\phi^{t}=\frac{1}{4}\left(\phi^{x}\right)^{2}$ (cf. conditions (a) and (a') in Remark 3.7) are associated with a fibration of $\Omega \times \mathbf{R}$ with graphs of affine -i.e., harmonic- functions as in Remark 4.2.

For the rest of this section, calibrations are always intended as Dirichlet calibrations for $F_{0}$, in the sense of Remark 3.7. We begin with a discussion of some one-dimensional examples. Of course, in these examples minimality can be easily checked by direct computations, and there would be no need for calibrations. Nevertheless, it is instructive to see what happens, and moreover some one-dimensional constructions are carried over to higher dimensions (cf. Paragraphs 4.10 and 4.12 ).

4.6. AfFine FUnCtion In ONE Dimension. - Let $\Omega$ be the open interval $(0, a)$ and let $u$ be the linear function $u(x):=\lambda x$, with $\lambda>0$. It is easy to see that $u$ is a Dirichlet minimizer of $F_{0}$ if and only if

$$
a \lambda^{2} \leq 1
$$

In this case a calibration is given by the piecewise constant vectorfield:

$$
\phi(x, t):= \begin{cases}\left(2 \lambda, \lambda^{2}\right) & \text { if } \frac{\lambda}{2} x \leq t \leq \frac{\lambda}{2}(x+a), \\ (0,0) & \text { otherwise. }\end{cases}
$$


Thus $\phi$ satisfies assumptions (a) and (a') of Remark 3.7, and vanishes outside a stripe of constant height (in grey in Figure 2 below, on the left) which is arranged so that (b) holds and $\operatorname{div} \phi$ vanishes (cf. Remark 2.8(b)), while (b') is trivially satisfied.

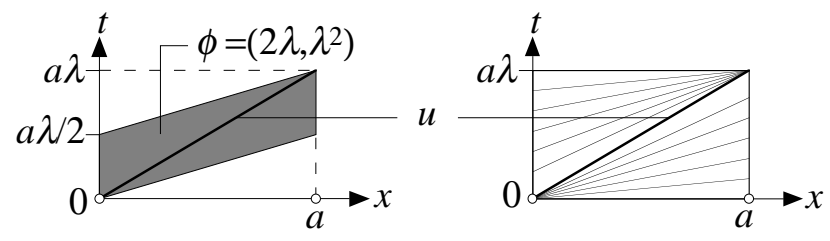

FiguRE 2

Another calibration is obtained by fibrating the rectangle $U=(0, a) \times(0, \lambda a)$ with affine functions as shown in Figure 2, on the right, and applying the construction of Remark 4.2:

$$
\phi(x, t):= \begin{cases}\left(2 \frac{t}{x},\left(\frac{t}{x}\right)^{2}\right) & \text { if } 0 \leq t \leq \lambda x \\ \left(2 \frac{\lambda a-t}{a-x},\left(\frac{\lambda a-t}{a-x}\right)^{2}\right) & \text { if } \lambda x \leq t \leq \lambda a .\end{cases}
$$

It remains to check that assumption (b) is satisfied, which happens if and only if (4.5) holds.

REMARK 4.7. - If $a \lambda^{2}<1$, then both calibrations described in the previous paragraph satisfy the strict inequality in assumption (b) of Remark 3.7, i.e.,

$$
\left|\int_{t_{1}}^{t_{2}} \phi^{x}(x, t) d t\right|<1 \quad \text { for every } x \in[0, a] \text { and every } t_{1}, t_{2} \in \mathbf{R} \text {. }
$$

By Remark 3.6, this shows that the function $u(x):=\lambda x$ is the unique Dirichlet minimizer of $F_{0}$ with $u(0)=0$ and $u(a)=\lambda a$.

4.8. Step function in one Dimension. - In Paragraph 4.6, in the limit case $a \lambda^{2}=1$ the linear function $u(x)=\lambda x$ and any step function of the form $u(x):=0$ for $0<x<c$ and $u(x):=\lambda a=\sqrt{a}$ for $c<x<a$ (with $0<c<a$ ) are both Dirichlet minimizers with the same boundary values. Hence both vectorfields (4.6) and (4.7) calibrate these step functions when $\lambda:=1 / \sqrt{a}$ (cf. Remark 3.6). Furthermore, it is easy to check that they also calibrate any step function $u$ given by $u(x):=0$ for $0<x<c$, and $u(x):=h$ for $c<x<a$ with $h \geq \sqrt{a}$.

REMARK 4.9. - When $a \lambda^{2}>1$ the linear function $u(x):=\lambda x$ is not a Dirichlet minimizer of $F_{0}$ (a step function is preferable), but it is still a Dirichlet $\bar{U}$ minimizer, when $U$ is the stripe of all points $(x, t)$ between the graph of $\lambda x-\frac{1}{4 \lambda}$ and $\lambda x+\frac{1}{4 \lambda}$. A calibration is given by $\phi(x, t):=\left(2 \lambda, \lambda^{2}\right)$. 


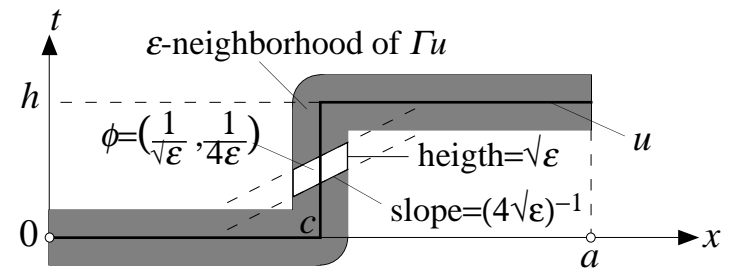

FiguRE 3

Conversely, when $h<\sqrt{a}$, the step function $u$ in Paragraph 4.8 is no longer a Dirichlet minimizer, but it is Dirichlet $\bar{U}$-minimizer when $U$ is an $\varepsilon$-neighbourhood of the complete graph of $u$ (in grey in Figure 3 ) and $\varepsilon$ satisfies $\frac{3}{2} \sqrt{\varepsilon}+2 \varepsilon \leq h$. A calibration is given by the piecewise constant vectorfield which vanishes outside the white parallelogram in Figure 3, and is equal to $\left(\frac{1}{\sqrt{\varepsilon}}, \frac{1}{4 \varepsilon}\right)$ inside.

4.10. HARMONIC FUNCTIONS IN DIMENSION $n$. - Let $u$ be a harmonic function on $\Omega$. Since $u$ is a Dirichlet minimizer of $\int_{\Omega}|\nabla u|^{2}$, it is natural to ask when it is also a Dirichlet minimizer of $F_{0}$. As pointed out by A. Chambolle, this happens when

$$
\operatorname{osc} u \cdot \sup |\nabla u| \leq 1
$$

where osc $u$ is the oscillation of $u$, namely the difference between the supremum $M$ and infimum $m$ of $u$ (over $\Omega$ ). In the one-dimensional case $n=1$ this condition reduces to (4.5).

A calibration can be constructed by analogy with (4.6); see Figure 4, on the left:

$$
\phi(x, t):= \begin{cases}\left(2 \nabla u(x),|\nabla u(x)|^{2}\right) & \text { if } \frac{1}{2}(u(x)+m) \leq t \leq \frac{1}{2}(u(x)+M) \\ (0,0) & \text { otherwise. }\end{cases}
$$

Another calibration can be obtained, as the one in (4.7), by embedding $u$ in a family of harmonic functions whose graphs fibrate the cylinder $\Omega \times[m, M]$. More precisely we take the functions $m+\lambda(u-m)$ and $M+\lambda(u-M)$ with $\lambda$ ranging in $[0,1]$ (see Figure 4, on the right), and then the construction of Remark 4.2 gives

$$
\phi(x, t):= \begin{cases}\left(2 \frac{t-m}{u(x)-m} \nabla u(x),\left(\frac{t-m}{u(x)-m}\right)^{2}|\nabla u(x)|^{2}\right) & \text { if } m \leq t \leq u(x), \\ \left(2 \frac{M-t}{M-u(x)} \nabla u(x),\left(\frac{M-t}{M-u(x)}\right)^{2}|\nabla u(x)|^{2}\right) & \text { if } u(x) \leq t \leq M .\end{cases}
$$

One easily checks that both vectorfields are divergence-free (see Remarks 2.8(b) and 4.2), and that assumptions (a) and (a') of Remark 3.7 are satisfied, assumption (b') is always trivially satisfied, while assumption (b) holds if an only if (4.8) holds. 


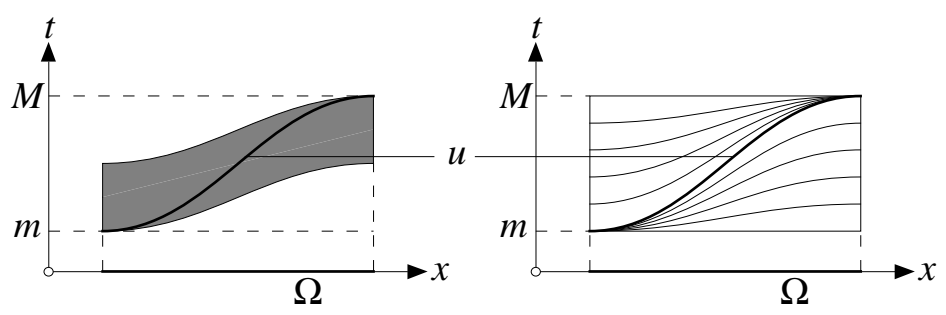

Figure 4

When (4.8) is not satisfied, $u$ is still a Dirichlet $\bar{U}$-minimizer of $F_{0}$, where $U$ is the set of all points $(x, t) \in \Omega \times \mathbf{R}$ which lie between the graph of $u(x)-(4|\nabla u(x)|)^{-1}$ and $u(x)+(4|\nabla u(x)|)^{-1}$; a calibration is given by $\phi(x, t):=$ $\left(2 \nabla u(x),|\nabla u(x)|^{2}\right)$.

REMARK 4.11. - If inequality (4.8) holds and $u$ is not affine, then the maximum principle implies that osc $u \cdot|\nabla u(x)|<1$ for every $x \in \Omega$, and therefore both calibrations constructed in the previous paragraph satisfy the strict inequality in assumption (b) of Remark 3.7. By Remark 3.6, this proves that the harmonic function $u$ is the only Dirichlet minimizer of $F_{0}$ with the same boundary values as $u$ (cf. Remark 4.7).

4.12. Step FUnCTION IN Dimension $n$. - Let $\Omega$ be a product of the form $(0, a) \times V$, where $V$ is a regular domain in $\mathbf{R}^{n-1}, n \geq 2$, and let $u$ be the step function given by $u(x):=0$ for $0<x_{1}<c$, and $u(x):=h$ for $c<x_{1}<a$, where $x_{1}$ denotes the first coordinate of $x, c \in(0, a)$, and $h \geq \sqrt{a}$.

Using the results in Paragraph 4.8 and a symmetrization argument, it is easy to see that $u$ is a Dirichlet minimizer of $F_{0}$. Calibrations can be constructed starting from the one-dimensional ones described in Paragraph 4.8. More precisely, we take the vectorfield on $\Omega \times \mathbf{R}$ which is parallel to the $\left(x_{1}, t\right)$-plane and is given by formula (4.6) (or even (4.7)) with $x$ replaced by $x_{1}$ and $\lambda:=1 / \sqrt{a}$.

REMARK 4.13. - The previous result can be restated by saying that a step function $u$ with jump of height $h$ along a hyperplane orthogonal to the direction $e$ is a Dirichlet minimizer whenever $h \geq \sqrt{a}$, where $a$ is the diameter of the projection of $\Omega$ along the $e$ axis. As in dimension one, any step function is a Dirichlet $\bar{U}$-minimizer, when $U$ is an $\varepsilon$-neighbourhood of the complete graph of $u$ and $\varepsilon$ satisfies $\frac{3}{2} \sqrt{\varepsilon}+2 \varepsilon \leq h$ (cf. Remark 4.9). However, unlike what happens in dimension one, $u$ may be a Dirichlet minimizer of $F_{0}$ even when the assumption $h \geq \sqrt{a}$ is not satisfied (cf. Paragraph 4.19 below).

4.14. Triple junction in the Plane. - Let $\Omega:=B(0, r)$ be the open disk in the plane with radius $r$ and centred at the origin, and let $\left(A_{1}, A_{2}, A_{3}\right)$ be the partition of $\Omega$ defined as follows: $A_{i}$ is the set of all $x \in \Omega$ of the form $x=(\rho \cos \theta, \rho \sin \theta)$, with $\frac{2}{3} \pi(i-1) \leq \theta<\frac{2}{3} \pi i$. Finally define $u:=a_{i}$ in each $A_{i}$, where $a_{1}<a_{2}<a_{3}$ are distinct constants.

Thus the singular set of $u$ is given by three line segments $S_{1,2}, S_{2,3}$, and $S_{3,1}$ meeting at the origin with equal angles (see Figure 5 , on the left), and it is 
well-known that this is a minimal network, in the sense that the corresponding partition $\left(A_{1}, A_{2}, A_{3}\right)$ is a Dirichlet minimizer of the "interface size" functional (see Remark 3.12). Therefore it is natural to conjecture that, when the values of the constant $a_{i}$ are sufficiently far apart, $u$ is a Dirichlet minimizer of $F_{0}$ too, that is, there is no convenience in removing part of the jump and taking a function with non-vanishing gradient.

We prove this conjecture by calibration. Inspired by the constructions described in Paragraphs 4.8 and 4.12 , we take $e_{ \pm}:=( \pm \sqrt{3} / 2,-1 / 2), \lambda>0$, and set

$$
\phi(x, t):= \begin{cases}\left(2 \lambda e_{-}, \lambda^{2}\right) & \text { if }\left|t-\frac{1}{2}\left(a_{1}+a_{2}\right)-\frac{\lambda}{2} x \cdot e_{-}\right|<\frac{1}{4 \lambda}, \\ \left(2 \lambda e_{+}, \lambda^{2}\right) & \text { if }\left|t-\frac{1}{2}\left(a_{2}+a_{3}\right)-\frac{\lambda}{2} x \cdot e_{+}\right|<\frac{1}{4 \lambda} \\ (0,0) & \text { otherwise. }\end{cases}
$$

Thus $\phi$ is piecewise constant, satisfies assumption (a) of Remark 3.7 by construction, and vanishes out of two slabs of constant height $\frac{1}{2 \lambda}$ (see Figure 5 , on the right). These slabs have been arranged in order to fulfill the following requirements:

(i) one slab is contained in $\Omega \times\left[a_{1}, a_{2}\right]$ and the other one in $\Omega \times\left[a_{2}, a_{3}\right]$, so that assumption (a') of Remark 3.7 is satisfied; it is possible to construct such slabs if we can choose $\lambda$ so that $a_{i+1}-a_{i} \geq \lambda r+\frac{1}{2 \lambda}$, that is, if

$$
a_{i+1}-a_{i} \geq \sqrt{2 r}
$$

(ii) the compatibility condition (2.5) is satisfied on the boundary of the slabs, so that $\phi$ is approximately regular and divergence-free (cf. Remark $2.8(\mathrm{~b}))$;

(iii) assumption (b') is satisfied for all points $x$ in $S_{1,2}$ and $S_{2,3}$, where $\nu_{u}$ coincides with $e_{-}$and $e_{+}$respectively.

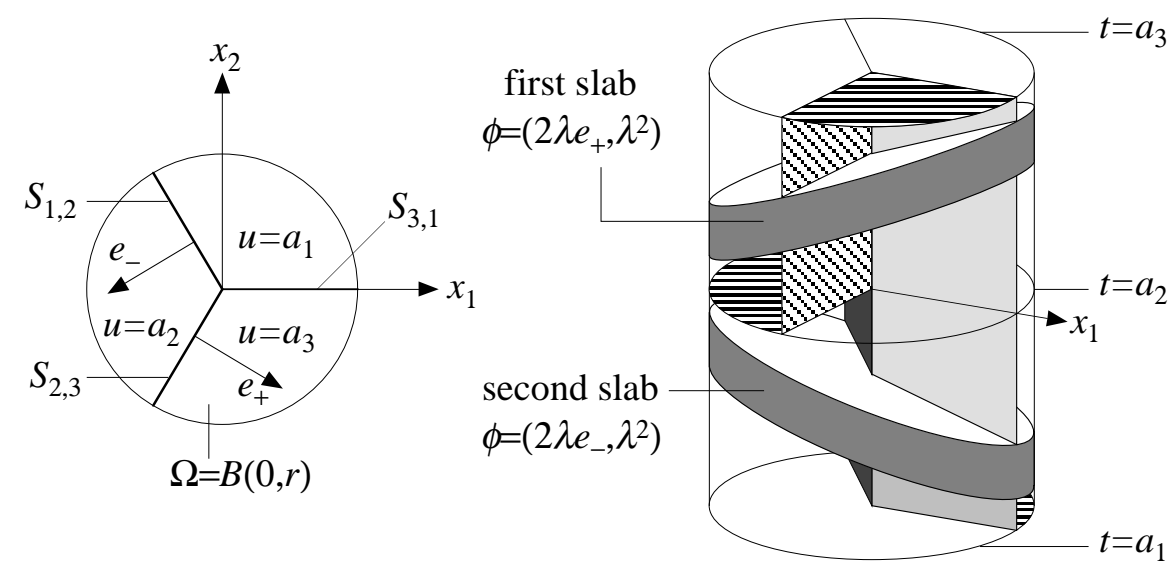

FIGURE 5

Moreover (b') holds also for $x$ in $S_{3,1}$, because $e_{-}+e_{+}=\nu_{u}$. One also checks that the integral $\int_{t_{1}}^{t_{2}} \phi^{x}(x, t) d t$ can be always written as a linear combination 
$\mu_{-} e_{-}+\mu_{+} e_{+}$with $\mu_{ \pm}$in $[0,1]$ (depending on $x, t_{1}, t_{2}$ ), and since $e_{+}$and $e_{-}$span an angle equal to $2 \pi / 3$, this implies that the integral has modulus not larger than 1. Thus (b) holds, too.

REMARK 4.15. - When $a_{2}-a_{1}$ (or $a_{3}-a_{2}$ ) is sufficiently small, $u$ is not a Dirichlet minimizer. More precisely, if $a_{2}-a_{1} \leq \frac{1}{3} \sqrt{r}$ (cf. (4.12)), a comparison function $v$ with the same boundary values as $u$ and such that $F(v)<F(u)$ is given, in polar coordinates, by

$$
v:= \begin{cases}\frac{1}{2}\left(a_{1}+a_{2}\right) & \text { if } 0 \leq \theta<\frac{4}{3} \pi \text { and } \rho \leq r-d, \\ \frac{1}{2}\left(a_{1}+a_{2}\right)+\frac{1}{2}\left(a_{1}-a_{2}\right) \frac{1}{d}(\rho-r+d) & \text { if } 0 \leq \theta<\frac{2}{3} \pi \text { and } \rho>r-d, \\ \frac{1}{2}\left(a_{1}+a_{2}\right)+\frac{1}{2}\left(a_{2}-a_{1}\right) \frac{1}{d}(\rho-r+d) & \text { if } \frac{2}{3} \pi \leq \theta<\frac{4}{3} \pi \text { and } \rho>r-d, \\ a_{3} & \text { if } \frac{4}{3} \pi \leq \theta<2 \pi,\end{cases}
$$

where $d:=\left(a_{2}-a_{1}\right) \sqrt{r}$ (we leave the computations to the reader).

4.16. Minimal partitions in Dimension $n$. - One can generalize the example of the triple junction, and conjecture the following: if a partition $\left(A_{1}, \ldots, A_{m}\right)$ of $\Omega$ is a Dirichlet minimizer of the "interface size" (see Remark 3.12) and $u$ is a function which takes a constant value $a_{i}$ on each $A_{i}$ (with $a_{1}<a_{2}<\ldots<a_{m}$ ), then $u$ is a Dirichlet minimizer of $F_{0}$ when the values $a_{i}$ are sufficiently far apart from each other. Unfortunately we can only prove this statement under two additional assumptions:

(i) the partition $\left(A_{1}, \ldots, A_{m}\right)$ is not only minimal, but admits a paired calibration in the sense of [34] and [11], namely there exist approximately regular, divergence-free vectorfields $\phi_{1}, \ldots, \phi_{m}$ on $\bar{\Omega}$ which satisfy assumptions (d) and (d') in Remark 3.12;

(ii) for $i=1, \ldots, m-1$ there exist Lipschitz functions $\psi_{i}: \bar{\Omega} \rightarrow \mathbf{R}$ which satisfy almost everywhere the first order equation

$$
\nabla \psi_{i} \cdot\left(\phi_{i+1}-\phi_{i}\right)=\frac{1}{2}\left|\phi_{i+1}-\phi_{i}\right|^{2} .
$$

Adding, if needed, a constant to $\psi_{i}$, we may also assume that

$$
\operatorname{osc} \psi_{i}=2\left\|\psi_{i}\right\|_{i} n f t y \text {. }
$$

For $i=1, \ldots, m-1$ we take slabs $U_{i}$, included in $\bar{\Omega} \times\left(a_{i}, a_{i+1}\right)$, of the form

$$
U_{i}=\left\{(x, t):\left|t-\frac{1}{2}\left(a_{i}+a_{i+1}\right)-\lambda_{i} \psi_{i}(x)\right|<\frac{1}{4 \lambda_{i}}\right\},
$$

where the constants $\lambda_{i}$ will be chosen below. Then we set (cf. (4.11))

$$
\phi(x, t):= \begin{cases}\left(2 \lambda_{i}\left(\phi_{i+1}(x)-\phi_{i}(x)\right), \lambda_{i}^{2}\left|\phi_{i+1}(x)-\phi_{i}(x)\right|^{2}\right) \\ & \text { if }(x, t) \in U_{i} \text { for some } i \\ (0,0) & \text { otherwise. }\end{cases}
$$


Taking into account assumption (d') in Remark 3.12 and the definition of the slabs $U_{i}$, one can easily check that assumptions (a), (a'), and (b') of Remark 3.7 are satisfied.

Let us check assumption (b). Taken $t_{1} \in\left[a_{i}, a_{i+1}\right]$ and $t_{2} \in\left[a_{j}, a_{j+1}\right]$ for some $i, j$, the integral $\int_{t_{1}}^{t_{2}} \phi^{x}(x, t) d t$ can be decomposed as the sum of the integrals on the (oriented) intervals $\left[t_{1}, a_{i+1}\right],\left[a_{i+1}, a_{j}\right]$, and $\left[a_{j}, t_{2}\right]$, and hence it can be written as

$$
\mu_{1}\left(\phi_{i+1}(x)-\phi_{i}(x)\right)+\left(\phi_{j}(x)-\phi_{i+1}(x)\right)+\mu_{2}\left(\phi_{j+1}(x)-\phi_{j}(x)\right)
$$

for suitable $\mu_{1}, \mu_{2} \in[0,1]$. But this sum can be reorganized as the difference between $\mu_{2} \phi_{j+1}(x)+\left(1-\mu_{2}\right) \phi_{j}(x)$ and $\mu_{1} \phi_{i}(x)+\left(1-\mu_{1}\right) \phi_{i+1}(x)$. Therefore its modulus is the distance between two points in the convex hull of the vectors $\phi_{1}(x), \ldots, \phi_{m}(x)$, which has diameter 1 because of assumption (d) in Remark 3.12 , and (b) is proved.

Since the vectorfields $\phi_{i}$ are divergence-free and approximately regular by assumption, $\phi$ is divergence-free and approximately regular within each slab (the approximate regularity of $\left(\phi^{x}, 0\right)$ is immediate, that of $\left(0, \phi^{t}\right)$ follows from Remark $2.3)$, as well as in the interior of the complement of the union of all slabs. Thus $\phi$ is divergence-free and approximately regular in $\Omega \times \mathbf{R}$ if (and only if) the compatibility condition (2.5) is satisfied on the boundary of each slab (cf. Remark $2.8(\mathrm{~b}))$, which reduces to equation (4.13).

Therefore we have constructed a calibration for $u$, provided that we can choose $\lambda_{i}$ so that the slabs $U_{i}$ are contained in $\Omega \times\left(a_{i}, a_{i+1}\right)$, that is,

$$
\frac{a_{i+1}-a_{i}}{2} \geq \lambda_{i}\left\|\psi_{i}\right\|_{\infty}+\frac{1}{4 \lambda_{i}}=\frac{\lambda_{i}}{2} \operatorname{osc} \psi_{i}+\frac{1}{4 \lambda_{i}}
$$

and this can be done as long as

$$
a_{i+1}-a_{i} \geq \sqrt{2 \operatorname{osc} \psi_{i}} \quad \text { for } i=1, \ldots, m-1 .
$$

REMARK 4.17. - A paired calibration for the partition $\left(A_{1}, A_{2}, A_{3}\right)$ described in Paragraph 4.14 is given by the constant vectorfields $\phi_{1}:=\frac{1}{6}(\sqrt{3}, 3)$, $\phi_{2}:=\frac{1}{6}(-2 \sqrt{3}, 0), \phi_{3}:=\frac{1}{6}(\sqrt{3},-3)$, and the linear functions $\psi_{1}$ and $\psi_{2}$ with gradients $\frac{1}{4}(-\sqrt{3},-1)$ and $\frac{1}{4}(\sqrt{3},-1)$ satisfy equation $(4.13)$; if we thus apply the construction of the previous paragraph, we obtain exactly the calibration described in Paragraph 4.14.

REMARK 4.18. - The first order equation (4.13) does not always admit solutions. For instance, since the derivative of $\psi_{i}$ along the integral curves of the vectorfield $\phi_{i+1}-\phi_{i}$ (i.e., the maximal solutions of the differential equation $\left.\dot{\gamma}=\phi_{i+1}(\gamma)-\phi_{i}(\gamma)\right)$ is always positive, when there exists a nontrivial closed integral curve within $\Omega$, (4.13) admits no solution. On the other hand, if $\phi_{i+1}-\phi_{i}$ is 
$C^{1}$ and nowhere vanishing, and all integral curves start and end at the boundary of $\Omega$ and intersect a fixed $(n-1)$-dimensional closed manifold $M$ in $\Omega$ which is transversal to the vectorfield $\phi_{i+1}-\phi_{i}$, i.e., a cross-section of the associated flow, then the method of characteristics provides a solution $\psi_{i}$ to (4.13) of class $C^{1}$.

However, such a strong requirement on $\phi_{i+1}-\phi_{i}$ is far from being necessary. Not only there may exist Lipschitz functions $\psi_{i}$ which satisfy (4.13) almost everywhere even if $\phi_{i+1}-\phi_{i}$ vanishes somewhere, but for the purposes of the previous construction we can even allow $\psi_{i}$ to be discontinuous along some integral curve $\gamma$ : in this case the boundary of the slab $U_{i}$ in (4.15) is not just the union of the graphs of $\lambda_{i} \psi_{i}+\frac{1}{2}\left(a_{1}+a_{2}\right)+\frac{1}{4 \lambda_{i}}$ and $\lambda_{i} \psi_{i}+\frac{1}{2}\left(a_{1}+a_{2}\right)-\frac{1}{4 \lambda_{i}}$, but there is an additional vertical piece contained in $\gamma \times \mathbf{R}$. Yet the compatibility condition (2.5) is satisfied there, and then $\phi$ is still divergence-free and approximately regular (cf. Remark 2.8(b)).

4.19. More on the STEP FUnCtion in the Plane. - Let $\Omega$ be the rectangle $(-a, a) \times(-b, b)$ in the plane, and let $\left(A_{1}, A_{2}\right)$ be the partition of $\Omega$ given by the sets of all points $x=\left(x_{1}, x_{2}\right)$ such that $x_{1}<0$ and $x_{1} \geq 0$ respectively. This partition is obviously minimal, and a paired calibration is $\phi_{1}:=(0,0), \phi_{2}:=(1,0)$. Setting $p_{+}:=(0, b)$ and $p_{-}:=(0,-b)$, another paired calibration is given by $\phi_{1}:=(0,0)$ and

$$
\phi_{2}(x):= \begin{cases}\left(-\sin \theta_{+}, \cos \theta_{+}\right) & \text {for } x \in B\left(p_{+}, b\right), \\ \left(\sin \theta_{-},-\cos \theta_{-}\right) & \text {for } x \in B\left(p_{-}, b\right), \\ (0,0) & \text { otherwise }\end{cases}
$$

where $\rho_{ \pm}, \theta_{ \pm}$are the polar coordinates around the points $p_{ \pm}$; see Figure 6 .

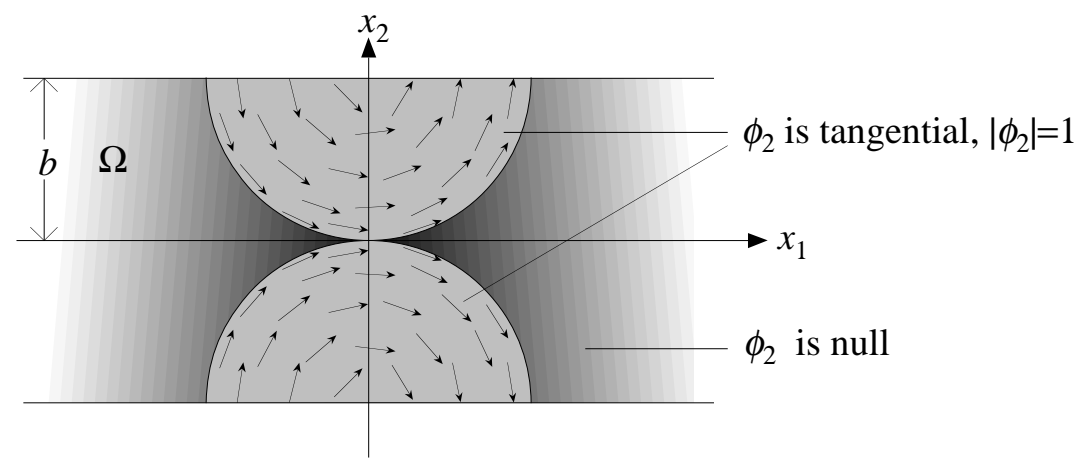

Figure 6

In this case, a function $\psi_{1}$ which satisfies (4.13) almost everywhere is given by

$$
\psi_{1}(x):= \begin{cases}\frac{1}{2}\left(\theta_{+}+\pi / 2\right) \rho_{+} & \text {for } x \in B\left(p_{+}, b\right), \\ \frac{1}{2}\left(\theta_{-}-\pi / 2\right) \rho_{-} & \text {for } x \in B\left(p_{-}, b\right) \\ 0 & \text { otherwise }\end{cases}
$$


and the construction in Paragraph 4.16, performed with some care because of the discontinuity of $\psi_{1}$ along the circles $\partial B\left(p_{ \pm}, b\right)$, yields a calibration for the step function $u$ which takes the value $a_{1}$ on $A_{1}$ and $a_{2}$ on $A_{2}$, provided that $a_{2}-a_{1} \geq \sqrt{\pi b}$. Note that the calibration obtained in this way is defined on the whole stripe $\mathbf{R} \times(-b, b)$ and does not depend on $a$. This extends the minimality result proved in Paragraph 4.12.

4.20. More ON THE TRIPLE JUNCTION. - Let us apply again the construction described in Paragraph 4.16 to the situation described in Paragraph 4.14 , with $\Omega$ replaced by an $\varepsilon$-neighbourhood of $S u$ within the ball $B(0, r)$ (in grey in Figure 7). As already noticed in Remark 4.17, a paired calibration for the partition $\left(A_{2}, A_{2}, A_{3}\right)$ is given by the constant vectorfields $\phi_{1}:=\frac{1}{6}(\sqrt{3}, 3)$, $\phi_{2}:=\frac{1}{6}(-2 \sqrt{3}, 0)$, and $\phi_{3}:=\frac{1}{6}(\sqrt{3},-3)$, but we can take solutions $\psi_{i}$ of $(4.13)$ such that $\left|\psi_{i}\right| \leq 2 \varepsilon$ on $\bar{\Omega}$, independently of the value of $r$. More precisely, we take the solution $\psi_{i}$ of (4.13) which takes the value 0 on the transversal set $M_{i}$ described in the Figure 7 for $i=1$ (the construction is similar for $i=2$ ).

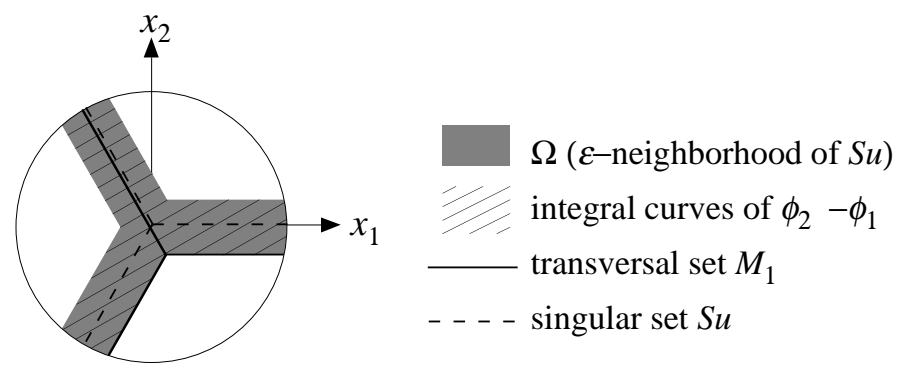

Figure 7

Therefore, if $a_{i+1}-a_{i} \geq 2 \sqrt{2 \varepsilon}$, the construction of Paragraph 4.16 yields a calibration of $u$ on $\bar{\Omega} \times \mathbf{R}$. Moreover this calibration can be extended to a calibration on an $\varepsilon$-neighbourhood of the complete graph of $u$ over the entire $B(0, r)$ by setting it equal to 0 where it is not yet defined. Clearly, this requires the slabs $U_{i}$ are contained in $B(0, r) \times\left(a_{i}+\varepsilon, a_{i+1}-\varepsilon\right)$ for $i=1$, 2 , that is

$$
a_{i+1}-a_{i} \geq 2 \varepsilon+2 \sqrt{2 \varepsilon} \text { for } i=1,2 .
$$

This shows that $u$ is a Dirichlet $\bar{U}$-minimizer of $F_{0}$ when $U$ is an $\varepsilon$-neighbourhood of the complete graph of $u$ within $B(0, r) \times \mathbf{R}$ and $\varepsilon$ satisfies (4.18). As expected, $\varepsilon$ does not depend on the size of the domain, but only on the relative distances of the values $a_{i}$.

\section{Applications to the complete Mumford-Shah functional}

In this section we focus on minimizers of the complete Mumford-Shah functional $F$ defined in (1.1) for $\alpha, \beta>0$, and calibrations will always be intended as 
in the sense of Theorem 3.4 and Definition 3.5. All the following examples are in dimension $n$.

5.1. Solutions of the Neumann Problem. - If we restrict $F$ to functions of class $W^{1,2}$, we obtain the strictly convex and coercive functional $\int_{\Omega}\left[|\nabla u|^{2}+\right.$ $\left.\beta(u-g)^{2}\right] d x$, and its unique minimizer $u$ is the solution of the Neumann problem

$$
\begin{cases}\Delta u=\beta(u-g) & \text { on } \Omega, \\ \partial_{\nu} u=0 & \text { on } \partial \Omega,\end{cases}
$$

where $\partial_{\nu}$ denotes the normal derivative. Thus it is natural to ask under which assumptions (on $g$ and $\beta$ ) $u$ is also a minimizer of $F$ on $S B V(\Omega)$. This question is akin to the minimality of harmonic functions for $F_{0}$ discussed in Paragraph 4.10, and following the same ideas we can construct an absolute calibration for $u$ provided that $u$ satisfy condition (5.3) below (cf. also Paragraph 5.4).

More precisely, we assume that $u$ is of class $C^{1}$ up to the boundary (this is always satisfied if $\partial \Omega$ is of class $C^{1, \varepsilon}$ for some $\varepsilon>0$ ), we denote the infimum and the supremum of $g$ by $m$ and $M$ respectively, and set

$$
A:=\left\{(x, t) \in \bar{\Omega} \times \mathbf{R}: \frac{u(x)+m}{2} \leq t \leq \frac{u(x)+M}{2}\right\},
$$

and (cf. (4.9))

$$
\phi^{x}(x, t):= \begin{cases}2 \nabla u(x) & \text { if }(x, t) \in A \\ 0 & \text { otherwise }\end{cases}
$$

Note that, by the maximum principle, $m \leq u \leq M$ on $\bar{\Omega}$, so that $\Gamma u$ is contained in $A$. Independently of the choice of $\phi^{t}$, we can already see that assumption (b) of Lemma 3.2 is satisfied if (cf. (4.8))

$$
\operatorname{osc} g \cdot \sup |\nabla u| \leq \alpha
$$

while assumption (b') is trivially satisfied, and $\phi$ has vanishing normal component on $\partial \Omega \times \mathbf{R}$ (because of (5.1)). Thus it remains to choose $\phi^{t}$ so that (a) and (a') hold, and $\phi$ is approximately regular and divergence-free.

Assumption (a') sets $\phi^{t}$ equal to $|\nabla u|^{2}-\beta(u-g)^{2}$ on the graph of $u$., while requiring that $\phi$ is divergence-free in the interior of $A$ yields

$$
\partial_{t} \phi^{t}=-\operatorname{div}_{x} \phi^{x}=-2 \Delta u=-2 \beta(u-g) .
$$

Integrating in $t$ we obtain that $\phi^{t}$ is given in $A$ by

$$
\begin{aligned}
\phi^{t} & =|\nabla u|^{2}-\beta(u-g)^{2}-2 \beta(u-g)(t-u) \\
& =|\nabla u|^{2}-\beta(t-g)^{2}+\beta(t-u)^{2} .
\end{aligned}
$$


Therefore assumption (a) of Lemma 3.2, namely $\phi^{t} \geq|\nabla u|^{2}-\beta(t-g)^{2}$, is clearly satisfied in $A$. Moreover $\phi$ is approximately continuous in $\bar{A}$ (this is trivial for the vectorfield $\left(\phi^{x}, 0\right)$, which is continuous, and follows from Remark 2.3 for $\left.\left(0, \phi^{t}\right)\right)$.

Moreover, $\phi$ is divergence-free in the complement of $A$ if we impose that $\partial_{t} \phi^{t}=$ 0 , that is, $\phi^{t}$ depends only on $x$, while the compatibility condition (2.5), which is required in order to have that $\phi$ is divergence-free on the entire $\Omega \times \mathbf{R}$ (cf. Remark $2.8(\mathrm{~b}))$, yields

$$
\phi^{t}= \begin{cases}-\beta\left(\frac{u+M}{2}-g\right)^{2}+\beta\left(\frac{u-M}{2}\right)^{2} & \text { for } t>\frac{u+M}{2}, \\ -\beta\left(\frac{u+m}{2}-g\right)^{2}+\beta\left(\frac{u-m}{2}\right)^{2} & \text { for } t<\frac{u+m}{2} .\end{cases}
$$

Finally one can easily check that $\phi$ is approximately regular also outside $A$ (see Remark 2.3) and satisfies condition (a) of Lemma 3.2 as well. Therefore $\phi$ is an absolute calibration for $u$, provided that $u$ satisfies (5.3).

REMARK 5.2. - If inequality (5.3) is strict, the calibration constructed in the previous paragraph satisfies the strict inequality in assumption (b) of Lemma 3.2, and by Remark 3.6 this proves that the solution $u$ of $(5.1)$ is the unique absolute minimizer of $F$.

REMARK 5.3. - The weaker inequality

$$
\operatorname{osc} u \cdot \sup |\nabla u| \leq \alpha
$$

which follows from (5.1) and (5.3) by the maximum principle, is not enough to guarantee the minimality of a solution $u$ of (5.1), not even the Dirichlet minimality. Take indeed $n:=1, \Omega:=(-a, a)$, and $g(x):=1$ for $x \geq 0 g(x):=-1$ for $x<0$. Then the solution to (5.1) can be computed explicitly:

$$
u(x):=\left[1-\frac{\cosh (\gamma(1-|x| / a))}{\cosh \gamma}\right] g(x),
$$

where $\gamma:=\sqrt{\beta} a$. Now we fix $\alpha$ so that (5.4) is satisfied, and precisely

$$
\alpha:=\operatorname{osc} u \cdot \sup |\nabla u|=2 \sqrt{\beta} \tanh \gamma\left[1-\frac{1}{\cosh \gamma}\right]
$$

and take the comparison function $v(x):=[1-1 / \cosh \gamma] g(x) ; v$ has the same boundary values as $u$, and a tedious but straightforward computation gives

$$
F(v)=2 \sqrt{\beta} \tanh \gamma-\frac{2 \sqrt{\beta}}{\cosh ^{2} \gamma}(\sinh \gamma-\gamma) \leq 2 \sqrt{\beta} \tanh \gamma=F(u) .
$$

Therefore $u$ satisfies condition (5.4) but is not a Dirichlet minimizer of $F$.

5.4. Solution of the Neumann problem for large $\beta$. - The construction in Paragraph 5.1 shows that the solution of the Neumann problem (5.1) is 
an absolute minimizer of $F$ provided that (5.3) holds. However, this condition is far from being necessary. In particular, for large values of the penalization parameter $\beta$, the absolute minimizer $u$ of $F$ is close to $g$, and therefore we expect that discontinuities should not be energetically convenient, at least for sufficiently regular $g$, and the solution $u$ of (5.1) should be the unique absolute minimizer of $F$.

We prove this fact by calibration under the assumption that $\Omega$ has boundary of class $C^{2}, g$ is of class $W^{2, p}$ for some $p>n$, and $\beta$ is larger than a certain $\beta_{0}$, specified in (5.15). Under these assumptions, $g$ belongs to $C^{1, \gamma}(\bar{\Omega})$ for $\gamma:=1-n / p$, and $u$ belongs to $C^{3, \gamma}(\Omega) \cap C^{1, \delta}(\bar{\Omega}) \cap W^{2, p}(\Omega)$ for every $\delta \in(0,1)$ by the standard regularity theory for Neumann problems (see, e.g., [39], Theorems 3.5, 3.16, and 3.17).

Fix a positive constant $\delta$ (to be properly chosen later), and take a smooth function $\sigma: \mathbf{R} \rightarrow[0,1]$, with support included in $[-2 \delta, 2 \delta]$ and identically equal to 1 in $[-\delta, \delta]$, so that $|\dot{\sigma}| \leq 2 / \delta$ (and then $\|\sigma\|_{1} \leq 4 \delta$ and $\|\dot{\sigma}\|_{\infty} \leq 2 / \delta$ ). Set

$$
\phi^{x}(x, t):=2 \sigma(t-u(x)) \nabla u(x) .
$$

To simplify the notation, in the following we simply write $\sigma$ and $\nabla u$ instead of $\sigma(t-u(x))$ and $\nabla u(x)$ (this must be kept into account when deriving), so that (5.5) becomes simply $\phi^{x}=2 \sigma \nabla u$.

It follows from (5.1) and (5.5) that $\phi$ has vanishing normal component at the boundary of $\Omega \times \mathbf{R}$, and $\phi^{x}=2 \nabla u$ on the graph of $u$. Assumption (a') in Lemma 3.2 prescribes the value of $\phi^{t}$ on the graph of $u$, and precisely

$$
\phi^{t}(x, u):=|\nabla u|^{2}-\beta(u-g)^{2} \quad \text { for all } x \in \bar{\Omega} .
$$

We impose now that $\phi$ is divergence-free, which reduces to

$$
\begin{aligned}
\partial_{t} \phi^{t}=-\operatorname{div}_{x} \phi^{x} & =-2 \sigma \Delta u+2 \dot{\sigma}|\nabla u|^{2} \\
& =-2 \beta \sigma(u-g)+2 \dot{\sigma}|\nabla u|^{2} .
\end{aligned}
$$

Identities (5.7) and (5.6) together determine $\phi^{t}$ everywhere.

Now we want to verify that assumption (a) of Lemma 3.2 holds, that is,

$$
\phi^{t} \geq \frac{1}{4}\left|\phi^{x}\right|^{2}-\beta(t-g)^{2} .
$$

Since the equality holds by construction on the graph of $u$, the full inequality is implied by the following inequalities on the derivatives of both sides of (5.8) with respect to $t$ :

$$
\begin{cases}\partial_{t} \phi^{t} \geq \frac{1}{2} \phi^{x} \partial_{t} \phi^{x}-2 \beta(t-g) & \text { for } t>u, \\ \partial_{t} \phi^{t} \leq \frac{1}{2} \phi^{x} \partial_{t} \phi^{x}-2 \beta(t-g) & \text { for } t<u .\end{cases}
$$

Let us consider the first inequality: by (5.5) and (5.7) it becomes

$$
-2 \beta \sigma(u-g)+2 \dot{\sigma}|\nabla u|^{2} \geq 2 \sigma \dot{\sigma}|\nabla u|^{2}-2 \beta(t-g),
$$


which is equivalent to

$$
\beta[(t-g)-\sigma(u-g)] \geq \dot{\sigma}(\sigma-1)|\nabla u|^{2} .
$$

When $u<t \leq u+\delta$ we have $\sigma=1$, and then (5.10) becomes $t-u \geq 0$, which is obviously true. When $t>u+\delta$, we have $(t-g)-\sigma(u-g) \geq \delta-\|u-g\|_{\infty}$ and $|\dot{\sigma}(\sigma-1)| \leq 2 / \delta$, and then (5.10) is implied by $\beta\left(\delta-\|u-g\|_{\infty}\right) \geq \frac{2}{\delta}\|\nabla u\|_{\infty}^{2}$. This inequality can be rewritten as

$$
\delta^{2}-\delta\|u-g\|_{\infty}-\frac{2}{\beta}\|\nabla u\|_{\infty}^{2} \geq 0
$$

and is satisfied for

$$
\delta \geq\|u-g\|_{\infty}+\sqrt{\frac{2}{\beta}}\|\nabla u\|_{\infty} .
$$

One checks in the same way that (5.11) implies the second inequality in (5.9) too. In other words, assumption (a) of Lemma 3.2 holds if (5.11) holds.

Assumption (b') of Lemma 3.2 is trivially satisfied because $S u$ is empty, while (5.5) and the estimate $\|\sigma\|_{1} \leq 4 \delta$ imply that assumption (b) of Lemma 3.2 is satisfied with strict inequality if $8 \delta\|\nabla u\|_{\infty}<\alpha$, that is,

$$
\delta<\frac{\alpha}{8\|\nabla u\|_{\infty}}
$$

Finally, we can find $\delta$ that satisfies both (5.11) and (5.12) if

$$
\|\nabla u\|_{\infty}\left(\sqrt{\beta}\|u-g\|_{\infty}+\sqrt{2}\|\nabla u\|_{\infty}\right)<\frac{\alpha}{8} \sqrt{\beta},
$$

and by Theorem 3.4 and Remark 3.6 we conclude that, if (5.13) is satisfied, then $u$ is the unique absolute minimizer of $F$.

Thus it remains to show that (5.13) holds for $\beta$ large enough. Note that $u$, being a solution of (5.1), depends on $\beta$, and there exist positive constants $K$ and $\bar{\beta}$ (depending on $\Omega$, but not on $g$ and $\beta$ ) such that for every $\beta \geq \bar{\beta}$ there holds

$$
\sqrt{\beta}\|u-g\|_{\infty}+\|\nabla u\|_{\infty} \leq K\|\nabla g\|_{W^{1, p}} .
$$

This estimate can be derived, for instance, from the interpolation inequality (3.1.59) of Theorem 3.1.22 in [28] (one has to replace $\lambda, u, \mathcal{A}$, and $\mathcal{B}$ with $\beta$, $u-g, \Delta$, and $\partial_{\nu}$ respectively, and recall that $\left.\Delta u=\beta(u-g)\right)$.

Estimate (5.14) shows that (5.13) holds for

$$
\beta>\beta_{0}:=\max \left\{\bar{\beta}, 2^{7} \alpha^{-2} K^{4}\|\nabla g\|_{W^{1, p}}^{4}\right\} .
$$

5.5. Characteristic Functions of Regular Sets. - If $g:=1_{E}$ is the characteristic function of a sufficiently regular compact subset $E$ of $\Omega$, then it is 
natural to conjecture that for large values of $\beta$ the minimizer of $F$ is $g$ itself. We prove this statement by calibration under the assumption that the boundary of $E$ is of class $C^{1,1}$ (cf. also Remark 5.6 below) and $\beta>\beta_{0}$, with $\beta_{0}$ defined by (5.22). Under these assumptions we also prove the uniqueness of the minimizer.

As in the previous paragraph, we first construct $\phi^{x}$. To this end, we take a Lipschitz vectorfield $\psi$ on $\bar{\Omega}$ which agrees on $\partial E$ with the inner normal of $\partial E$, is supported on a neighbourhood of $\partial E$ which is relatively compact in $\Omega$, and satisfies $|\psi| \leq 1$ everywhere. For instance, we can use that $\partial E$ is locally a graph, which yields a trivial extension of the normal vectorfield on a small neighbourhood of each point, and then use a partition of unity to paste together these different extensions. Now we set

$$
\phi^{x}(x, t):=\sigma(t) \psi(x) \text { for all } x \in \bar{\Omega}, t \in \mathbf{R},
$$

where $\sigma: \mathbf{R} \rightarrow[0,2 \alpha]$ is a function of class $C^{1}$, supported in [0,1], with integral equal to $\alpha$, and such that $|\dot{\sigma}(t)| \leq 16 \alpha$ for $t \in[0,1], \sigma(t):=t^{2}$ for $t \in[0,1 / 8]$, $\sigma(t):=(1-t)^{2}$ for $t \in[7 / 8,1]$.

We see that, independently of the choice of $\phi^{t}$, the vectorfield $\phi$ has vanishing normal component at the boundary of $\Omega$, and satisfies assumptions (b) and (b') of Lemma 3.2. Since $\phi^{x}$ vanishes for $t=0$ and for $t=1$, and therefore on the graph of $g$, requiring that $\phi$ satisfies assumption (a') yields

$$
\phi^{t}(x, g(x)):=0 \quad \text { for } \mathcal{L}^{n} \text {-a.e. } x \in \Omega,
$$

while requiring that $\phi$ is divergence-free yields (cf. (5.16))

$$
\partial_{t} \phi^{t}=-\operatorname{div}_{x} \phi^{x}=-\sigma \operatorname{div}_{x} \psi .
$$

Conditions (5.17) and (5.18) together determine $\phi^{t}$.

Note that $\phi$ is approximately regular: this is trivial for the vectorfield $\left(\phi^{x}, 0\right)$, which is continuous, and follows from Remark 2.3 for $\left(0, \phi^{t}\right)$ (even though $\phi^{t}$ is discontinuous on $\partial E \times \mathbf{R}$ and where $\operatorname{div}_{x} \psi$ is).

To show that $\phi$ is an absolute calibration it remains thus to verify assumption (a) of Lemma 3.2, namely

$$
\phi^{t} \geq \frac{1}{4}\left|\phi^{x}\right|^{2}-\beta(t-g)^{2}=\frac{1}{4} \sigma^{2}|\psi|^{2}-\beta(t-g)^{2} .
$$

Since the equality holds by construction on the graph of $g$ (cf. (5.17)), it is enough that $\partial_{t} \phi^{t}$ satisfies the inequality

$$
\partial_{t} \phi^{t}:=-\sigma \operatorname{div}_{x} \psi>\frac{1}{2} \sigma \dot{\sigma}|\psi|^{2}-2 \beta(t-g) \text { for } t>g(x)
$$

and the opposite inequality for $t<g(x)$. Inequality (5.20) is clearly satisfied for $t>1$, since $\sigma(t)=0$. If $g(x)=0$ and $0<t \leq 1,(5.20)$ is implied by

$$
-\sigma\left\|\operatorname{div}_{x} \psi\right\|_{\infty}>\frac{1}{2} \sigma|\dot{\sigma}|-2 \beta t .
$$


In turn, (5.21) reduces for $0<t<1 / 8$ to

$$
-t^{2}\left\|\operatorname{div}_{x} \psi\right\|_{\infty}>t^{3}-2 \beta t
$$

which is satisfied for $\beta>\frac{1}{16}\left\|\operatorname{div}_{x} \psi\right\|_{\infty}+\frac{1}{128}$, while, for $1 / 8 \leq t \leq 1,(5.21)$ follows from

$$
-2 \alpha\left\|\operatorname{div}_{x} \psi\right\|_{\infty}>16 \alpha^{2}-\frac{1}{4} \beta,
$$

which is satisfied for $\beta>8 \alpha\left\|\operatorname{div}_{x} \psi\right\|_{\infty}+64 \alpha^{2}$. Therefore (5.20) holds for

$$
\beta>\beta_{0}:=\max \left\{\frac{1}{16}\left\|\operatorname{div}_{x} \psi\right\|_{\infty}+\frac{1}{128}, 16 \alpha\left\|\operatorname{div}_{x} \psi\right\|_{\infty}+64 \alpha^{2}\right\} .
$$

The same condition implies also the opposite inequality for $t<g(x)$. This concludes the proof that $\phi$ calibrates $g$.

To prove that $g$ is the unique minimizer of $F$, we first notice that the strict inequality in (5.20) implies the strict inequality in (5.19) for $t>g(x)$, and of course we have the strict inequality for $t<g(x)$, too. In other words, the inequality in assumption (a) of Lemma 3.2 is strict for all $t \neq g(x)$. Now, if $u$ is another minimizer, $\phi$ must calibrate $u$, too (cf. Remark 3.6), and in particular it must satisfy assumption (a') of Lemma 3.2 for $u$, which means that the inequality in assumption (a) is an equality for $t=u(x)$. Therefore we conclude that $u(x)=g(x)$ for $\mathcal{L}^{n}$-a.e. $x$ in $\Omega$.

REMARK 5.6. - If $g:=1_{E}$ is the characteristic function of a set $E$ relatively compact in $\Omega$ and $u:=g$ minimizes $F$, then the set $E$ minimizes in particular $\mathcal{F}(A):=F\left(1_{A}\right)=\alpha \mathcal{H}^{n-1}\left(\partial_{*} A\right)+\beta|A \triangle E|$ among all sets $A$ with finite perimeter in $\Omega$. Hence the regularity theory for minimal perimeters yields that that, in dimension $n \leq 7, E$ must be of class $C^{1, \gamma}$ for every $\gamma<1$, while in dimension two it must be of class $C^{1,1}$ (see, e.g., [5], Theorem 4.7.4). Thus the regularity on $g$ required in the previous paragraph is optimal in dimension two, and close to optimal for $3 \leq n \leq 7$.

REMARK 5.7. - If $g$ is the characteristic function of a set $E$ of class $C^{1,1}$ which is not relatively compact in $\Omega$, then the result of Paragraph 5.5 can be generalized as follows: $u:=g$ is a minimizer of $F$ for large values of $\beta$ provided that $\partial E$ is orthogonal to $\partial \Omega$ (which is assumed to be sufficiently smooth). An absolute calibration can be constructed as in the previous paragraph, one has only to choose $\psi$ so that it is tangent to the boundary of $\Omega$.

Notice that this orthogonality requirement is necessary: indeed it is easily proved that, given a minimizer of the functional $\alpha \mathcal{H}^{n-1}\left(\Omega \cap \partial_{*} A\right)+\beta|A \triangle E|$ among all finite perimeter sets $A$ in $\Omega$, its boundary is orthogonal to $\partial \Omega$.

We conclude this section with some remarks on the gradient flow associated with the (homogeneous) Mumford-Shah functional.

5.8. Gradient Flow for the Mumford-Shah functional. - A gradient flow for $F_{0}$ with respect to the $L^{2}$-metric can be defined in a variational way by 
time discretization, following the minimizing movements approach developed in [19], [4], [27]. Given an initial datum $u_{0} \in L^{2}(\Omega)$ and a discretization step $\delta>0$, we set $u_{\delta, 0}:=u_{0}$ and define inductively $u_{\delta, j}$ for $j=1,2, \ldots$ as any minimizer of

$$
F_{0}(u)+\frac{1}{\delta} \int_{\Omega}\left(u-u_{\delta, j-1}\right)^{2} d x
$$

among all functions $u$ in $S B V(\Omega)$ - with or without prescribed boundary values, according to the boundary condition (Dirichlet or Neumann) imposed on the flow. Then we define $u_{\delta}: \Omega \times[0,+\infty) \rightarrow \mathbf{R}$ by $u_{\delta}(x, t):=u_{\delta, j}$ for $t:=j \delta$, and by linear interpolation for $t \in(j \delta,(j+1) \delta)$, and call gradient flow with initial datum $u(x, 0)=u_{0}(x)$ any possible limit of $u_{\delta}$ as $\delta \rightarrow 0$ along any sequence. Note that the flow may be not unique, as even $u_{\delta}$ is not uniquely defined.

REMARK 5.9. - If $u_{0}$ belongs to $W^{1,2}(\Omega)$, and the minimization of (5.23) is restricted a priori to the functions $u$ in $W^{1,2}(\Omega)$, then $F_{0}(u)$ is just the usual Dirichlet integral, and it can be proved (cf. [4], Example 2.1) that the previous construction yields a unique flow which agrees with the solution of the heat equation

$$
\partial_{t} u=\Delta u \quad \text { on } \Omega \times(0,+\infty)
$$

with initial datum $u(x, 0)=u_{0}(x)$ and boundary conditions - Neumann or Dirichlet - according to the boundary conditions imposed in the minimization of (5.23).

The previous remark and the result of Paragraph 5.4 suggest that for a smooth initial datum $u_{0}$, the gradient flow associated with $F_{0}$ is just the solution of the heat equation. To prove this, however, we need some additional information on the minima of $F$ for large $\beta$.

5.10. Improved estimates on the SOlution of (5.1). - Under the regularity assumptions on $\Omega$ and $g$ given in Paragraph 5.4, if $\Delta g \in L^{\infty}(\Omega)$ and $\partial_{\nu} g=0$ on $\partial \Omega$, then the solution $u$ to the Neumann problem (5.1) satisfies

$$
\|\Delta u\|_{\infty} \leq\|\Delta g\|_{\infty}
$$

and an improved version of estimate (5.14):

$$
\beta\|u-g\|_{\infty}+\|\nabla u\|_{\infty} \leq K\|\Delta g\|_{\infty}
$$

with $K$ depending on $\Omega$, but not on $g$ and $\beta$. In particular condition (5.13) of Paragraph 5.4 holds for

$$
\beta>\beta_{0}:=\max \left\{1,2^{7} \alpha^{-2} K^{4}\|\Delta g\|_{\infty}^{4}\right\},
$$

and in that case $u$ is the unique absolute minimizer of $F$. 
To prove (5.24) and (5.25), we first notice that the function $v:=g+\varepsilon$ is a super-solution of (5.1) as long as $\varepsilon \geq \beta^{-1}\|\Delta g\|_{\infty}$, in the sense that

$$
\begin{cases}\Delta v \leq \beta(v-g) & \text { on } \Omega, \\ \partial_{\nu} v \leq 0 & \text { on } \partial \Omega .\end{cases}
$$

Thus $u$ is (a.e.) smaller than $g+\varepsilon$ on $\Omega$. Similarly, $g-\varepsilon$ is a sub-solution, and then

$$
\|u-g\|_{\infty} \leq \frac{1}{\beta}\|\Delta g\|_{\infty}
$$

which, in view of (5.1), implies (5.24).

Now, $u$ solves the equation $\Delta u=f$ with Neumann boundary conditions and $f:=\beta\left(u-g\right.$ ), and well-known estimates (cf. [39], Theorem 3.16) give $\|\nabla u\|_{\infty} \leq$ $K\|f\|_{\infty}$ for a suitable constant $K$ depending on $\Omega$, but not on $f$. Together with (5.27), this implies (5.25).

5.11. Solution of the Dirichlet problem for large $\beta$. - Assume that $\Omega$ and $g$ satisfy the regularity assumptions of Paragraph 5.4, $\Delta g$ belongs to $L^{\infty}(\Omega)$, and $g_{0}$ is a function in $W^{2, p}(\Omega)$, and consider the solution $u$ to the Dirichlet problem

$$
\begin{cases}\Delta u=\beta(u-g) & \text { on } \Omega, \\ u=g_{0} & \text { on } \partial \Omega .\end{cases}
$$

Then $u$ belongs to $C^{3, \gamma}(\Omega) \cap C^{1, \delta}(\bar{\Omega}) \cap W^{2, p}(\Omega)$ for every $\delta \in(0,1)$ (see, e.g., [39], Theorems 3.5, 3.16, and 3.17). We claim that if $g_{0}=g$ on $\partial \Omega$ and $\beta$ is sufficiently large, then $u$ is the unique Dirichlet minimizer of $F$ with boundary value $g_{0}$.

This claim can be proved by the same calibration constructed in Paragraph 5.4 , provided that estimate (5.14) is suitably replaced. To this end, we notice that $v:=g+\varepsilon$ is a super-solution of (5.28) for $\varepsilon \geq \beta^{-1}\|\Delta g\|_{\infty}$, in the sense that

$$
\begin{cases}\Delta v \leq \beta(v-g) & \text { on } \Omega, \\ v \geq g_{0} & \text { on } \partial \Omega\end{cases}
$$

(we use here that $g=g_{0}$ on $\partial \Omega$ ), and, similarly, $g-\varepsilon$ is a sub-solution. As in the previous paragraph, we deduce that $\beta\|u-g\| \leq\|\Delta g\|_{\infty}$, and hence (cf. (5.24))

$$
\|\Delta u\|_{\infty} \leq\|\Delta g\|_{\infty}
$$

Let us consider now the function $w:=u-g_{0}$; since it solves $\Delta w=f-\Delta g_{0}$ with Dirichlet boundary conditions $w=0$ on $\partial \Omega$, and $f:=\beta(u-g)$, well-known estimates for solutions of Dirichlet problems (see, e.g., [39], Theorem 3.16) give $\|\nabla w\|_{\infty} \leq K\left(\|f\|_{\infty}+\left\|\nabla g_{0}\right\|_{C^{0, \gamma}}\right)$. Together with the estimate on $\|u-g\|_{\infty}$, this implies (cf. (5.25))

$$
\beta\|u-g\|_{\infty}+\|\nabla u\|_{\infty} \leq K\left(\|\Delta g\|_{\infty}+\left\|\nabla g_{0}\right\|_{C^{0, \gamma}}\right),
$$


with a possibly different $K$. In particular, condition (5.13) of Paragraph 5.4 is satisfied for

$$
\beta>\beta_{0}:=\max \left\{1,2^{7} \alpha^{-2} K^{4}\left(\|\Delta g\|_{\infty}+\left\|\nabla g_{0}\right\|_{C^{0, \gamma}}\right)^{4}\right\},
$$

and in that case $u$ is the unique Dirichlet minimizer of $F$ with boundary values $u=g_{0}$.

5.12. Gradient Flow with smooth initial datum. - Assume that $\Omega$ has boundary of class $C^{2}, u_{0} \in W^{2, p}(\Omega)$ for some $p>n, \Delta u_{0} \in L^{\infty}(\Omega)$, and $\partial_{\nu} u_{0}=0$ on $\partial \Omega$. Then the gradient flow for $F_{0}$ with Neumann boundary conditions and initial datum $u(x, 0)=u_{0}(x)$ constructed in Paragraph 5.8 is unique, and agrees with the solution of the heat equation.

In virtue of Remark 5.9, this claim is an immediate consequence of the following fact: when

$$
\delta<\delta_{0}:=\left[\max \left\{1,2^{7} \alpha^{-2} K^{4}\left\|\Delta u_{0}\right\|_{\infty}^{4}\right\}\right]^{-1},
$$

then, for every integer $j$, every minimizer of (5.23) belongs to $W^{1,2}(\Omega)$. In other words, the solution of the Neumann problem (5.1) with $\beta:=1 / \delta$ and $g:=u_{\delta, j-1}$ is the unique minimizer of (5.23).

To prove this fact, it suffices to verify that the assumptions of Paragraph 5.10 are satisfied for every $j$, and precisely: $u_{\delta, j-1} \in W^{2, p}(\Omega), \Delta u_{\delta, j-1} \in L^{\infty}(\Omega)$, $\partial_{\nu} u_{\delta, j-1}=0$ on $\partial \Omega$, and inequality (5.26) holds with $\beta:=1 / \delta$ and $g:=u_{\delta, j-1}$. The last requirement follows from the choice of $\delta$ and the chain of inequalities

$$
\left\|\Delta u_{0}\right\|_{\infty}:=\left\|\Delta u_{\delta, 0}\right\|_{\infty} \geq\left\|\Delta u_{\delta, 1}\right\|_{\infty} \geq\left\|\Delta u_{\delta, 2}\right\|_{\infty} \geq \ldots
$$

which are implied by (5.24). The $W^{2, p}$ regularity of $u_{\delta, j}$ follows from the corresponding regularity of $u_{\delta, j-1}$, as remarked at the beginning of Paragraph 5.4.

REMARK 5.13. - The conclusion of the previous paragraph also holds for the gradient flow with Dirichlet boundary conditions. More precisely, if $\Omega$ has boundary of class $C^{2}$ and $u_{0}$ is of class $W^{2, p}(\Omega)$, with $\Delta u_{0} \in L^{\infty}(\Omega)$, then the gradient flow for $F_{0}$ with initial datum $u(x, 0)=u_{0}(x)$ on $\Omega$ and Dirichlet boundary condition $u(x, t)=u_{0}(x)$ on $\partial \Omega \times[0,+\infty)$ constructed in Paragraph 5.8 is unique, and agrees with the solution of the heat equation (with same initial datum and boundary conditions). The proof is essentially the same as in the Neumann case, and relies on the estimates given Paragraph 5.11 (in particular one has to apply (5.28) with $g=u_{\delta, j-1}$ and $\left.g_{0}=u_{0}\right)$.

REMARK 5.14. - If we drop the assumption $\partial_{\nu} u_{0}=0$ on $\partial \Omega$ in Paragraph 5.12 , the proof breaks down because we can no longer estimate $\left\|\Delta u_{\delta, j-1}\right\|_{\infty}$ by $\left\|\Delta u_{0}\right\|_{\infty}$, but we do not know if the conclusion on the gradient flow still holds. A similar problem occurs with the statement in Remark 5.13 if we replace the Dirichlet boundary condition $u(x, t)=u_{0}(x)$ on $\partial \Omega$ with a different one. 
REMARK 5.15. - If $u_{0}:=1_{E}$ is the characteristic function of a compact subset of $\Omega$ with boundary of class $C^{1,1}$, then the gradient flow for $F_{0}$ with Neumann (or Dirichlet) boundary conditions constructed in Paragraph 5.8 is unique, and is just given by $u(x, t):=u_{0}(x)$ on $\Omega \times[0,+\infty)$. This follows immediately from Paragraph 5.5.

REMARK 5.16. - The conclusions of Paragraph 5.12 and Remark 5.15 support the following general conjecture: if the initial datum $u_{0}$ is smooth out of a smooth singular set $S u$, which is compact in $\Omega$, then, at least for small times, the associated gradient flow should be unique and should keep the singular set of $u(\cdot, t)$ equal to $S u_{0}$, while the function $u$ evolves in $\Omega \backslash S u_{0}$ according to the heat equation (with Neumann conditions on $S u$ ). This conjecture has been proved in the one-dimensional case in [26] (with a slightly different definition of gradient flow for $F_{0}$ ). The general case will be studied in [35] using the calibration method.

\section{Appendix}

In this section we prove some technical lemmas stated in Section 2. We follow the notation of Section 2 .

LEMmA 6.1. - Let $\Omega$ be an open subset of $\mathbf{R}^{n}$ whose boundary is the graph of a Lipschitz function $f: \mathbf{R}^{n-1} \rightarrow \mathbf{R}$, and let $\phi$ be a bounded vectorfield on $\bar{\Omega}$ which has bounded support and satisfies condition (2.4) with $M:=\partial \Omega$. Then there exists a sequence of vectors $y_{j}$ such that $y_{j} \rightarrow 0, \partial \Omega+y_{j} \subset \Omega$ for every $j$, and

$$
\lim _{j \rightarrow \infty} \phi\left(x+y_{j}\right) \cdot \nu_{\partial \Omega}(x)=\phi(x) \cdot \nu_{\partial \Omega}(x) \quad \text { for } \mathcal{H}^{n-1} \text {-a.e. } x \in \partial \Omega .
$$

Proof. Let $S$ be the set of all vectors $y \in \mathbf{R}^{n}$ such that $\partial \Omega+y \subset \Omega$. For every $r>0$, let $S_{r}:=S \cap B(0, r)$, and consider the double integral

$$
\int_{S_{r}}\left[\int_{\partial \Omega}\left|(\phi(x+y)-\phi(x)) \cdot \nu_{\partial \Omega}(x)\right| d \mathcal{H}^{n-1}(x)\right] \frac{d y}{r^{n}} .
$$

If we invert the order of integration, condition (2.4) means that the inner integral (over $S_{r}$ ) tends to 0 as $r \rightarrow 0$ for $\mathcal{H}^{n-1}$-a.e. $x \in \partial \Omega$. Then (6.2) converges to 0 by the dominated convergence theorem (recall that $\phi$ is bounded and has bounded support).

As $\partial \Omega$ is a graph of a Lipschitz function, the set $S$ contains an open cone with vertex in 0 . Then the measure of $S_{r}$ is larger than $a r^{n}$ for some fixed $a>0$, and therefore we can choose $y_{r} \in S_{r}$ so that the value of the inner integral in (6.2) is smaller than the double integral divided by $a$, and then converges to 0 as $r \rightarrow 0$.

In other words, $\phi\left(x+y_{r}\right) \cdot \nu_{\partial \Omega}(x)$ converge to $\phi(x) \cdot \nu_{\partial \Omega}(x)$ in $L^{1}\left(\partial \Omega, \mathcal{H}^{n-1}\right)$, and then it suffices to choose a subsequence $y_{j}$ which yields pointwise convergence for $\mathcal{H}^{n-1}$-a.e. $x \in \partial \Omega$. 
Proof of Lemma 2.4. - (Sketch) We divide the proof in several steps.

STEP 1. Assume that $\phi$ belong to $C_{c}^{1}\left(\mathbf{R}^{n}, \mathbf{R}^{n}\right)$. In this case formula (2.6) is well-known - see, e.g., [25], Theorem 2.10, or [6], formula (3.87) in Theorem 3.87.

STEP 2. Assume that $\phi$ is an approximately regular vectorfield on $\mathbf{R}^{n}$ with compact support and that $\operatorname{div} \phi \in L^{\infty}\left(\mathbf{R}^{n}\right)$. Let $\psi_{\varepsilon}(x):=\varepsilon^{-n} \psi(x / \varepsilon)$ be a standard radially symmetric regularizing kernel of class $C_{c}^{\infty}$, and take $\phi_{\varepsilon}:=\phi * \psi_{\varepsilon}$. Thus formula (2.6) holds for each $\phi_{\varepsilon}$ by Step 1, and it only remains to check that we can pass to the limit as $\varepsilon \rightarrow 0$. The convergence of the first integral in the right-hand side of (2.6) follows from the fact that the functions $\operatorname{div} \phi_{\varepsilon}$ are bounded in $L^{\infty}$ and converge to $\operatorname{div} \phi$ a.e. in $\Omega$. Since $\phi$ is approximately regular, the maps $\phi_{\varepsilon} \cdot \nu_{M}$ converge to $\phi \cdot \nu_{M} \mathcal{H}^{n-1}$-a.e. on any Lipschitz surface $M$, and then also on any rectifiable set $M$. In particular this implies the convergence of the second integral in the right-hand side of (2.6). The same argument also applies to the left-hand side, provided that we use the coarea formula (cf. [6], Theorem 3.40, or [23], Theorem 4.5.9(13)) to re-write that integral as

$$
\int_{\Omega} \phi_{\varepsilon} \cdot D u=\int_{\mathbf{R}}\left[\int_{M_{t}} \phi \cdot \nu_{M_{t}} d \mathcal{H}^{n-1}\right] d t
$$

where $M_{t}$ is the measure theoretic boundary in $\Omega$ of the sublevel $\{u<t\}$.

STEP 3. If $\phi$ is a compactly supported, approximately regular vectorfield on a neighbourhood of $\bar{\Omega}$ with $\operatorname{div} \phi$ in $L^{\infty}$, we reduce to Step 2 using a suitable cut-off function.

STEP 4. Assume that $\Omega$ is the subgraph of a Lipschitz function $f: \mathbf{R}^{n-1} \rightarrow \mathbf{R}$, and $\phi$ is a compactly supported, approximately regular vectorfield on $\bar{\Omega}$ with $\operatorname{div} \phi \in L^{\infty}(\Omega)$. We take a sequence of vectors $y_{j}$ as in Lemma 6.1, and set $\phi_{j}(x):=\phi\left(x+y_{j}\right), u_{j}(x):=u\left(x+y_{j}\right)$. By Step 3, formula (2.6) holds with $\phi$ and $u$ replaced by $\phi_{j}$ and $u_{j}$, and it remains to check that we can pass to the limit as $j \rightarrow+\infty$. The convergence is immediate for all integrals in (2.6) but the last one. In this case, it suffices to notice that the functions $\phi_{j} \cdot \nu_{\partial \Omega}$ are uniformly bounded and converge to $\phi \cdot \nu_{\partial \Omega} \mathcal{H}^{n-1}$-a.e. on $\partial \Omega$ (by the choice of the vectors $y_{j}$ ), while the traces of $u_{j}$ on $\partial \Omega$ converge to the trace of $u$ in $L^{1}\left(\partial \Omega, \mathcal{H}^{n-1}\right)$ (because the functions $u_{j}$ converge to $u$ in variation, or, alternatively, because the $L^{1}$-norm of the difference of the traces is controlled, up to a constant which does not depend on $j$, by $|D u|\left(\Omega \backslash\left(\bar{\Omega}-y_{j}\right)\right)$, which clearly tends to zero).

STEP 5. To prove the general case, we use a locally finite partition of unity consisting of compactly supported smooth functions to reduce to Step 4.

Proof of Lemma 2.6. - We first prove that $\operatorname{div} \phi=f$ on $\Omega \backslash S_{0}$. Since the problem is local, it is enough to show that $\operatorname{div} \phi=f$ on every bounded open set $U$ with $\bar{U} \subset \Omega \backslash S_{0}$ and such that $U \backslash S_{1}$ has two connected components $U^{+}$and $U^{-}$with Lipschitz boundary. As $\phi$ is approximately regular on $\bar{U}^{ \pm}$, we can apply formula (2.6) with $\Omega$ replaced by $U^{ \pm}$and $u \in C_{c}^{\infty}(U)$ : as the integrals on $U \cap S_{1}$ 
cancel out, we are left with $\int_{U} \phi \cdot \nabla u d x=-\int_{U} f u d x$. By the arbitrariness of $u$, we deduce that $\operatorname{div} \phi=f$ on $U$.

We prove now that $\operatorname{div} \phi=f$ on $\Omega$. Since $\mathcal{H}^{n-1}\left(S_{0}\right)=0$, the $(1,1)$-capacity of $S_{0}$ is zero (see [22], Section 5.6.3), and therefore there exists a sequence of functions $\sigma_{j}$ in $C^{\infty}(\bar{\Omega})$ such that $0 \leq \sigma_{j} \leq 1$ in $\Omega$, and $\sigma_{j}=0$ in a neighbourhood of $S_{0}$, and $\sigma_{j} \rightarrow 1$ strongly in $W^{1,1}(\Omega)$.

Take now an arbitrary function $u \in C_{c}^{\infty}(\Omega)$. Then the functions $\sigma_{j} u$ belong to $C_{c}^{\infty}\left(\Omega \backslash S_{0}\right)$ and, since $\operatorname{div} \phi=f$ on $\Omega \backslash S_{0}$, we have $\int_{\Omega} \phi \cdot \nabla\left(\sigma_{j} u\right) d x=$ $-\int_{\Omega} f \cdot\left(\sigma_{j} u\right) d x$. Moreover the functions $\sigma_{j} u$ converge to $u$ strongly in $W^{1,1}(\Omega)$, and therefore $\int_{\Omega} \phi \cdot \nabla u d x=-\int_{\Omega} f u d x$, which concludes the proof.

Proof of Lemma 2.10. - By a monotone class argument it is enough to prove (2.9) for $\phi$ of the form $\phi(x, t):=\rho(t) \psi(x)$, with $\rho: \mathbf{R} \rightarrow \mathbf{R}$ and $\psi=$ $\left(\psi^{x}, \psi^{t}\right): \Omega \rightarrow \mathbf{R}^{n} \times \mathbf{R}$ of class $C_{c}^{\infty}$. Let $\sigma$ be the primitive of $\rho$ vanishing at $-\infty$. Then we have

$$
\begin{aligned}
\int_{\Omega \times \mathbf{R}} \phi \cdot D 1_{u} & =-\int_{\Omega \times \mathbf{R}} \operatorname{div} \phi 1_{u} d x \\
& =-\int_{\Omega}\left[\int_{-\infty}^{u}\left(\rho \operatorname{div}_{x} \psi^{x}+\dot{\rho} \psi^{t}\right) d t\right] d x \\
& =-\int_{\Omega}\left[\sigma(u) \operatorname{div}_{x} \psi^{x}+\rho(u) \psi^{t}\right] d x
\end{aligned}
$$

As $u$ belongs to $S B V(\Omega)$, the chain-rule for $B V$-functions (see, e.g., [6], Theorem 3.96) gives

$$
D[\sigma(u)]=\rho(u) \nabla u \cdot \mathcal{L}^{n}+\left[\sigma\left(u^{+}\right)-\sigma\left(u^{-}\right)\right] \nu_{u} \cdot \mathcal{H}^{n-1}\llcorner S u
$$

Therefore (6.3) implies

$$
\begin{aligned}
\int_{\Omega \times \mathbf{R}} \phi \cdot D 1_{u}= & \int_{\Omega}\left[\rho(u) \psi^{x} \cdot \nabla u-\rho(u) \psi^{t}\right] d x \\
& +\int_{S u}\left[\sigma\left(u^{+}\right)-\sigma\left(u^{-}\right)\right] \psi^{x} \cdot \nu_{u} d \mathcal{H}^{n-1}
\end{aligned}
$$

which, together with (2.8), gives (2.9) in the case $\phi(x, t):=\rho(t) \psi(x)$.

Proof of Lemma 2.12. - We set $w:=1_{u}-1_{v}$ on $\Omega \times \mathbf{R}$. Then $w$ belongs to $B V(\Omega \times \mathbf{R})$ and $D w=\nu_{\Gamma u} \cdot \mathcal{H}^{n}\left\llcorner\Gamma u-\nu_{\Gamma v} \cdot \mathcal{H}^{n}\llcorner\Gamma v\right.$.

Let us consider the inner trace of $w$ on $\partial U$. First of all we decompose $\partial U$ as the disjoint union of $(\Omega \times \mathbf{R}) \cap \partial U$ and $(\partial \Omega \times \mathbf{R}) \cap \partial U$. For every $C^{\infty}$ vectorfield $\psi$ on $\Omega \times \mathbf{R}$ with compact support we apply formula (2.6) of Lemma 2.4 with $\Omega$ 
and $\phi$ replaced by $U$ and $\psi$, respectively, and we obtain

$$
\begin{aligned}
-\int_{U} w \operatorname{div} \psi d x= & \int_{U} \psi \cdot D w+\int_{\partial U} w \psi \cdot \nu_{\partial U} d \mathcal{H}^{n} \\
= & \int_{\Gamma u \cap U} \psi \cdot \nu_{\Gamma u} d \mathcal{H}^{n}-\int_{\Gamma v \cap U} \psi \cdot \nu_{\Gamma v} d \mathcal{H}^{n} \\
& +\int_{\partial U} w \psi \cdot \nu_{\partial U} d \mathcal{H}^{n} .
\end{aligned}
$$

On the other hand, by the definition of distributional derivative we have also

$$
\begin{aligned}
-\int_{\Omega \times \mathbf{R}} w \operatorname{div} \psi d x & =\int_{\Omega \times \mathbf{R}} \psi \cdot D w \\
& =\int_{\Gamma u} \psi \cdot \nu_{\Gamma u} d \mathcal{H}^{n}-\int_{\Gamma v} \psi \cdot \nu_{\Gamma v} d \mathcal{H}^{n}
\end{aligned}
$$

Due to the particular structure of $U$ and the assumption on the complete graphs of $u$ and $v$, the function $w$ vanishes a.e. on $(\Omega \times \mathbf{R}) \backslash U$. This fact, together with (6.4) and (6.5), implies that the inner trace of $w$ on $(\Omega \times \mathbf{R}) \cap \partial U$ satisfies

$$
w \nu_{\partial U}=1_{\Gamma u} \nu_{\Gamma u}-1_{\Gamma v} \nu_{\Gamma v} \quad \mathcal{H}^{n} \text {-a.e. on }(\Omega \times \mathbf{R}) \cap \partial U
$$

Therefore $w$ belongs to $L^{1}\left((\Omega \times \mathbf{R}) \cap \partial U, \mathcal{H}^{n}\right)$ and

$$
\int_{(\Omega \times \mathbf{R}) \cap \partial U} w \phi \cdot \nu_{\partial U} d \mathcal{H}^{n}=\int_{\Gamma u \cap \partial U} \phi \cdot \nu_{\Gamma u} d \mathcal{H}^{n}-\int_{\Gamma v \cap \partial U} \phi \cdot \nu_{\Gamma v} d \mathcal{H}^{n}
$$

Now, the trace of $w$ on $\partial \Omega \times \mathbf{R}$ is the difference of the characteristic functions of the traces of $u$ and $v$ on $\partial \Omega$, and therefore it belongs to $L^{1}\left(\partial \Omega \times \mathbf{R}, \mathcal{H}^{n}\right)$ and vanishes $\mathcal{H}^{n}$-a.e. on $(\partial \Omega \times \mathbf{R}) \backslash \partial U$. As $\nu_{\partial U}=\left(\nu_{\partial \Omega}, 0\right)$ on $(\partial \Omega \times \mathbf{R}) \cap \partial U$, this implies

$$
\begin{aligned}
\int_{(\partial \Omega \times \mathbf{R}) \cap \partial U} w \phi & \cdot \nu_{\partial U} d \mathcal{H}^{n}=\int_{\partial \Omega \times \mathbf{R}} w \phi^{x} \cdot \nu_{\partial \Omega} d \mathcal{H}^{n} \\
& =\int_{\partial \Omega}\left[\int_{v}^{u} \phi^{x}(x, t) d t\right] \cdot \nu_{\partial \Omega} d \mathcal{H}^{n-1} .
\end{aligned}
$$

Since the inner trace of $w$ on $\partial U$ belongs to $L^{1}\left(\partial U, \mathcal{H}^{n}\right)$, we apply formula (2.6) of Lemma 2.4 with $\Omega$ and $u$ replaced by $U$ and $w$, respectively, and get

$$
\int_{\Gamma u \cap U} \phi \cdot \nu_{\Gamma u} d \mathcal{H}^{n}-\int_{\Gamma v \cap U} \phi \cdot \nu_{\Gamma v} d \mathcal{H}^{n}=-\int_{\partial U} w \phi \cdot \nu_{\partial U} d \mathcal{H}^{n}
$$

Identity (2.10) follows now from (6.6), (6.7), and (6.8). 


\section{References}

1. G. Alberti, L. Ambrosio, X. Cabré: On a long-standing conjecture of E. De Giorgi: symmetry in $3 \mathrm{~d}$ for general nonlinearities and a local minimality property. Acta Appl. Math., to appear (downloadable from http://cvgmt.sns.it/papers).

2. G. Alberti, G. Bouchitté, G. Dal Maso: The calibration method for the Mumford-Shah functional. C. R. Acad. Sci. Paris Sér. I Math. 329 (1999), 249-254.

3. L. Ambrosio: Existence theory for a new class of variational problems. Arch. Rational Mech. Anal. 111 (1990), 291-322.

4. L. Ambrosio: Movimenti minimizzanti. Rend. Accad. Naz. Sci. XL Mem. Mat. (5) 19 (1995), 191-246.

5. L. Ambrosio: Corso introduttivo alla teoria geometrica della misura ed alle superfici minime. Appunti dei Corsi Tenuti da Docenti della Scuola (Notes of courses given by teachers at the School). Scuola Normale Superiore, Pisa, 1997.

6. L. Ambrosio, N. Fusco, D. Pallara: Functions of bounded variation and free discontinuity problems. Oxford Mathematical Monographs. Oxford Science Publications, Oxford, 1999.

7. G. Anzellotti: Traces of bounded vector-fields and the divergence theorem. Unpublished preprint, Dipartimento di Matematica, Università di Trento, Trento, 1983.

8. G. Bellettini, A. Coscia, G. Dal Maso: Compactness and lower semicontinuity properties in $S B D(\Omega)$. Math. Z. 228 (1998), 337-351.

9. A. Bonnet, G. David: Cracktip as a global Mumford-Shah minimizer. Preprint Univ. Paris Sud, Orsay, 2000 (downloadable from http://www.math.u-psud.fr/biblio/pub/2000/).

10. G. Bouchitté, A. Chambolle: Paper in preparation.

11. K.A. Brakke: Minimal cones on hypercubes. J. Geom. Anal. 1 (1991), 329-338.

12. K.A. Brakke: Soap films and covering spaces. J. Geom. Anal. 5 (1995), 445-514.

13. K.A. Brakke: Numerical solution of soap film dual problems. Experiment. Math. 4 (1995), 269-287.

14. P. Celada: Minimum problems on $S B V$ with irregular boundary datum. Rend. Sem. Mat. Univ. Padova 98 (1997), 193-211.

15. A. Chambolle: Convex representation for lower semicontinuous functionals in $L^{1}$. J. Convex Anal., to appear (downloadable from http://www. ceremade.dauphine.fr/cadrepub.html).

16. G. Dal Maso: The calibration method for free discontinuity problems. Proceedings of the third european congress of mathematics (Barcelona, 2000), to appear.

17. G. Dal Maso, M.G. Mora, M. Morini: Local calibrations for minimizers of the MumfordShah functional with rectilinear discontinuity sets. J. Math. Pures Appl. (9) 79 (2000), 141-162.

18. G. Dal Maso, J.-M. Morel, S. Solimini: A variational method in image segmentation: existence and approximation results. Acta Math. 168 (1992), 89-151.

19. E. De Giorgi: New problems on minimizing movements. Boundary value problems for partial differential equations and applications (dedicated to E. Magenes), 81-98, edited by J.-L. Lions and C. Baiocchi. Research Notes in Applied Mathematics, 29. Masson, Paris, 1993.

20. E. De Giorgi, L. Ambrosio: Un nuovo funzionale del calcolo delle variazioni. Atti Accad. Naz. Lincei Rend. Cl. Sci. Fis. Mat. Natur. (8) 82 (1988), 199-210.

21. E. De Giorgi, M. Carriero, A. Leaci: Existence theorem for a minimum problem with free discontinuity set. Arch. Rational Mech. Anal. 108 (1989), 195-218.

22. L.C. Evans, R.F. Gariepy: Measure theory and fine properties of functions. Studies in Advanced Mathematics. CRC Press, Boca Raton, 1992.

23. H. Federer: Geometric measure theory. Grundlehren der mathematischen Wissenschaften, 153. Springer-Verlag, Berlin-New York, 1969. Reprinted in the series Classics in Mathematics. Springer-Verlag, Berlin-Heidelberg, 1996.

24. H. Federer: Real flat chains, cochains and variational problems. Indiana Univ. Math. J. 24 (1974-75), 351-407. 
25. E. Giusti: Minimal surfaces and functions of bounded variation. Monographs in Mathematics, 80. Birkhäuser Boston, Boston, 1984.

26. M. Gobbino: Gradient flow for the one-dimensional Mumford-Shah functional. Ann. Scuola Norm. Sup. Pisa Cl. Sci. (4) 27 (1998), 145-193.

27. M. Gobbino: Minimizing movements and evolution problems in Euclidean spaces. Ann. Mat. Pura Appl. (4) 176 (1999), 29-48.

28. A. Lunardi: Analytic semigroups and optimal regularity in parabolic problems. Progress in Nonlinear Differential Equations and their Applications, 16. Birkhäuser Verlag, Basel, 1995.

29. M. Miranda: Superfici cartesiane generalizzate ed insiemi di perimetro localmente finito sui prodotti cartesiani. Ann. Scuola Norm. Sup. Pisa Cl. Sci. (3) 18 (1964), 515-542.

30. M.G. Mora, M. Morini: Local calibrations for minimizers of the Mumford-Shah functional with a regular discontinuity sets. Ann. Inst. H. Poincaré, Anal. Non Linéaire, to appear (downloadable from http://www.sissa.it/fa/publications).

31. J.-M. Morel, S. Solimini: Variational methods in image segmentation. Progress in Nonlinear Differential Equations and their Applications, 14. Birkhäuser Boston, Boston, 1995.

32. F. Morgan: Calibrations and new singularities in area-minimizing surfaces: a survey. Variational methods (Paris, 1988), 329-342, edited by H. Berestycki et al. Progr. Nonlinear Differential Equations Appl., 4. Birkhäuser Boston, Boston, 1990.

33. F. Morgan: Area-minimizing currents bounded by higher multiples of curves. Rend. Circ. Mat. Palermo (2) 33 (1984), 37-46.

34. F. Morgan, G. Lawlor: Paired calibrations applied to soap films, immiscible fluids, and surfaces or networks minimizing other norms. Pacific J. Math. 166 (1994), 55-83.

35. M. Morini: Global calibrations for the non-homogeneous Mumford-Shah functional. Paper in preparation.

36. D. Mumford, J. Shah: Boundary detection by minimizing functionals. IEEE Conference on Computer Vision an Pattern Recognition, San Francisco, 1985.

37. D. Mumford, J. Shah: Optimal approximation by piecewise smooth functions and associated variational problems. Comm. Pure Appl. Math. 42 (1989), 577-685.

38. J.E. Taylor: The structure of singularities in soap-bubble-like and soap-film-like minimal surfaces. Ann. of Math. (2) 103 (1976), 489-539.

39. G.M. Troianiello: Elliptic differential equations and obstacle problems. The University Series in Mathematics. Plenum Press, New York, 1987.

40. B. White: The least area bounded by multiples of a curve. Proc. Amer. Math. Soc. 90 (1984), 230-232.

\section{Giovanni Alberti}

Dipartimento di Matematica Università di Pisa

via Buonarroti 2, 56127 Pisa

ITALY

e-mail: alberti@dm.unipi.it

\section{Guy Bouchitté}

UFR des Sciences et Techniques Université de Toulon et du Var BP 132, 83957 La Garde Cedex FRANCE

e-mail: bouchitte@univ-tln.fr

Gianni Dal Maso

S.I.S.S.A.

via Beirut 4, 34014 Trieste

ITALY

e-mail: dalmaso@sissa.it 